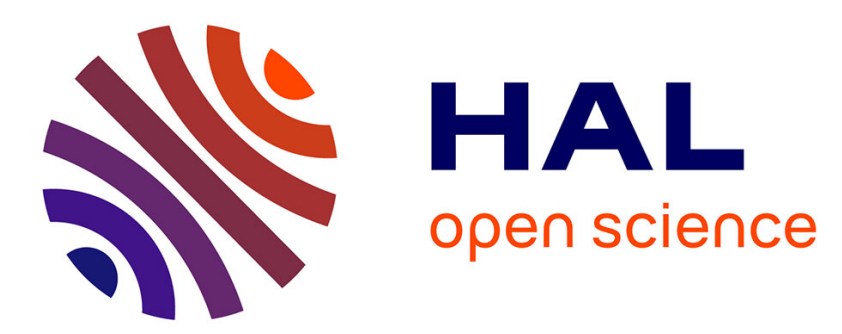

\title{
On the mathematical modeling of vole populations spatial dynamics via transport equations on a graph
}

Carlotta Donadello, Thi Nhu Thao Nguyen, Ulrich Razafison

\section{To cite this version:}

Carlotta Donadello, Thi Nhu Thao Nguyen, Ulrich Razafison. On the mathematical modeling of vole populations spatial dynamics via transport equations on a graph. Applied Mathematics and Computation, 2021, 396. hal-02908168v2

\section{HAL Id: hal-02908168 https://hal.science/hal-02908168v2}

Submitted on 14 Jan 2021

HAL is a multi-disciplinary open access archive for the deposit and dissemination of scientific research documents, whether they are published or not. The documents may come from teaching and research institutions in France or abroad, or from public or private research centers.
L'archive ouverte pluridisciplinaire HAL, est destinée au dépôt et à la diffusion de documents scientifiques de niveau recherche, publiés ou non, émanant des établissements d'enseignement et de recherche français ou étrangers, des laboratoires publics ou privés. 


\title{
On the mathematical modeling of vole populations spatial dynamics via transport equations on a graph
}

\author{
Carlotta Donadello Thi Nhu Thao Nguyen $\quad$ Ulrich Razafison \\ Laboratoire de Mathématiques de Besançon, CNRS UMR 6623, \\ Université Bourgogne Franche-Comté, \\ 16 route de Gray, 25030 Besançon Cedex, France
}

\begin{abstract}
In this paper, we propose two models to describe the spatial dynamic of vole populations together with their finite volumes discretization. The models are based on age-structured transport equations set on a graph. The local evolution of the population occurs at the nodes, while transmission between nodes represents spatial dynamics and is a gradual process in the first model, an instantaneous one in the second. The parameters in the reproduction and mortality rates allows to reproduce the characteristics of different landscapes.
\end{abstract}

Keywords: Age-structured population models, Transport equations, Dispersal, Finite-volume schemes, Numerical simulations

AMS Classification: 35Q92, 92B05, 92D25, 65M08

\section{Introduction}

Small rodents, as voles, are cornerstone species in most temperate ecosystems as their presence is necessary to the survival of a variety of predators. At the same time, rodents can be vectors of serious disease transmissible to humans, and cause significant loss in agricultural production and storage.

Direct observations performed in several different countries suggest that the populations dynamics of voles is characterized by large density fluctuations related to dispersal, i.e. a collective movement in which a significant part of the juveniles in the colony leave to initiate new settlements. A huge literature is devoted to the mathematical modeling of such populations, with no hope to be exhaustive we defer the interested reader to [16, 20] and the references therein. Most of the literature only focus on a two variables dynamics (time and age or time and space) but the observations suggest that the evolution depend on the three of them. The papers [4] and [2], and the monograph [21] use different PDE techniques to deal with three variables models. In the present paper we follow a partially different idea, inspired from [13]. In [13], Marilleaux, Lang and Giraudoux reproduced the first colonization dynamics observed from 1998 to 2010 in the Romanche Valley (Hautes Alpes, France) by an hybrid ODE-Multi Agents model structured as follows : the valley area is decomposed into squared cells of side length $100 \mathrm{~m}$, so that at a first approximation the evolution of the population inside each of the cells can be described by an ODE (no spatial dynamics). As soon as the total population in a cell reaches a fixed threshold value, which is a fraction of the cell capacity, dispersal occurs, i.e. some of the juvenile voles "leave" the cell, and aggregate into a migrating vole agent with a specific dynamics also depending on 
the topography and landscape characteristics of the neighbouring cell agents. In the following we refer to this model as MLG.

The advantage of this approach over the purely PDE one is that simulations are fast and their results are visualized in a format which is accessible to a non specialized audience. At the same time, the description of the population dynamics inside cells is relatively rough, and the set of parameters for which computational results match empirical observations is not organized in a continuum, which makes it difficult to predict the behaviour of the model under perturbations.

In the present paper we exploit the idea of decoupling the local population evolution from its spatial dynamics to construct a simple but effective PDE model structured on a graph whose nodes correspond to the cells in the MLG model. Together with the abstract model, we propose its discretization in the form of a suitable finite volumes numerical scheme. Then we run several simulations showing that our model captures many of the features of voles population dynamics as they have been observed in real life. Finally, in view of the construction of an enhanced hybrid model combining a refined PDE description of the evolution in the cells and the Multi Agent approach to represent dispersal, we also consider a model in which the dynamics inside the nodes is splitted from the dynamics on the graph by using different time scales (instantaneous dispersal).

The paper is organized as follows: in Section 2 we describe the models and motivate our assumptions on the basis of observed population characteristics; In Section 3 we present the corresponding finite volumes numerical schemes and validate them by comparison to explicitely computed exact solutions. Section 4 collects some numerical simulations on simple graphs, which show that the models reproduce the qualitative features of the population dynamics, while Section 5 contains a short summary and the perspective directions of this project.

\section{Description of our models}

In this section we propose two models to describe the spatial dynamics of a voles population structured in age. Both models write as transport equations on graph.

Voles' sex ratio at birth is 1-1, [3, 15], so that it is enough to model the female population, which is structured into three age classes: young voles, whose age $a$ varies between 0 and $A_{1}$, juveniles, $a \in\left[A_{1}, A_{2}\right]$, and adults, $a>A_{2}$. The same observations suggest that $A_{1} \approx 18$ days and $A_{2} \approx 56$ days, but for simplicity we assume the normalization $A_{2}=3 A_{1}$. Since most voles die out of predation, disease or starvation, at a first approximation we could neglect aging when describing the adult population: in particular it is not necessary to consider a decline of fertility over age. Nevertheless, to avoid non realistic results when performing simulations on a large time horizon (e.g. 4 years), we define $A_{3}$ as the maximal age that a vole individual can attain (different values will be taken into account) and we set our model so that individuals of age larger than $A_{3}$, if any, do not participate any more in the evolution of the system. As numerical values for age transitions, we fix $A_{1}=1, A_{2}=3$ and $A_{3}=20$.

We partition the natural space into squared cells of side length $100 \mathrm{~m}, V_{i}, i=1, \ldots, n_{\nu}$. We measure the distances between cells centers and, based on observations, we estimate the average time needed by a vole to cover such distances. Then we define a finite weighted graph $\Gamma=(\mathscr{E}, V)$, whose set of nodes $V=\left\{V_{1}, \ldots, V_{n_{v}}\right\}$ represents the cells, while the set of its edges $\mathscr{E}$ consists of the triples $\left(V_{i}, V_{j}, \ell_{i j}\right)$ where $V_{i} \neq V_{j}$ belong to $V$ (no loops are allowed) and $\ell_{i j}>0$ is the travel time between the two vertices. For simplicity we will always assume that $\Gamma$ is not directed, therefore $\ell_{i j}=\ell_{j i}$. In general it is possible to assume that the graph is complete, i.e. that there exists an edge joining any given pair of nodes. From the modeling point of view this is compatible with the fact that some individuals (long range dispersers) can be recaptured at observation sites more than $5 \mathrm{Km}$ apart from each other. 
Each node $V_{i}$ is endowed with an initial boundary value problem representing the evolution of the density of voles in $V_{i}, \rho_{i}$, with respect to the time and age variables $t$ and $a$,

$$
\begin{aligned}
& \rho_{i}: \quad \mathbb{R}^{+} \times \mathbb{R}^{+} \rightarrow \mathbb{R}^{+} \\
& (t, a) \quad \mapsto \quad \rho_{i}(t, a) .
\end{aligned}
$$

The total number of voles in $V_{i}$ at time $t$ is given by the integral

$$
\Phi_{i}(t)=\int_{0}^{A_{3}} \rho_{i}(t, a) \mathrm{d} a .
$$

The spatial dynamics of the population is represented by transmissions of density from one node to the others. The local spatial dynamics, related to foraging activities is neglected. While most individuals spend almost all of their lives in burrows, where they also breed, some of the juveniles have a significant spatial dynamics during dispersals. The mechanism triggering dispersal is still not completely understood and it is most probably related to the interaction of several factors (see for instance [1, 2, 3, 5, 7, 9, 10, 18, 19] and references therein). However, for modeling purposes, it is reasonable to link stress conditions to overcrowding, and say that dispersal begins when the total number of individuals in a colony reaches a threshold value, fixed as a fraction of the carrying capacity of the environment, $R$. New colonies most often appear in a radius of a few hundreds meters around the original one, but individuals might disperse over more than $5 \mathrm{Km}$. Topography, in particular relief, drives the dispersal direction, and during this phase voles' mortality is very high.

We define a stochastic matrix $\mathbf{K}$ of size $n_{\nu} \times n_{\nu}$. Its coefficients $k_{i j}$ fix which fraction of voles leaving the node $V_{i}$ would head toward $V_{j}$. Since no loops are allowed, we set $k_{i i}=0$ for any $i \in\left\{1, \ldots, n_{\nu}\right\}$. At a modeling level we can imagine that $k_{i j}$ depends, for example, on the height or the landscapes in $V_{i}$ and $V_{j}$, therefore in general $k_{i j} \neq k_{j i}$. From the modeling point of view it makes sense to consider a time dependent matrix K. An example in this direction is given in Section 4.2.2, but for the simplicity of the presentation, we keep K constant for the moment.

Assume that at time $\bar{t}$ some of the voles initially at $V_{i}$ disperse. Since voles age and die during dispersal but cannot reproduce, the evolution of voles leaving $V_{i}$ at time $\bar{t}$ and age $a$ and heading toward $V_{j}$, is described by the ODE problem

$$
\left\{\begin{array}{l}
\partial_{s} u(s)=-\mathfrak{d}_{d i s} u(s), \\
u(0)=k_{i j} \rho_{i}(\bar{t}, a)
\end{array}\right.
$$

where $\mathfrak{d}_{d i s}$ represents mortality during dispersal. The estimated travel time from $V_{i}$ to $V_{j}$ is the weight $\ell_{i j}$, therefore the density of voles of age $a+\ell_{i j}$ reaching $V_{j}$ at time $\bar{t}+\ell_{i j}$ is

$$
k_{i j} \rho_{i}(\bar{t}, a) \exp \left(-\ell_{i j} \mathfrak{d}_{d i s}\right)=K_{i j} \rho_{i}(\bar{t}, a)
$$

Observe that the matrix $\mathscr{K}=\left(K_{i j}\right) \in \mathbb{R}^{n_{v} \times n_{v}}$ is not stochastic, not symmetric and depends on time if and only if $\mathbf{K}$ does.

\subsection{A model with gradual dispersal (GD model)}

We always assume that a dispersal begins from the node $V_{i}$ whenever its total population $\Phi_{i}$ reaches the threshold $R_{i}$. In the first model, the dispersal lasts for a time $\eta>0$ after the moment at which $\Phi_{i}$ passes again below $R_{i}$ and the departure rate of juveniles during dispersal is $c / \eta$, where $c>0$ is a parameter to be chosen. Notice that $R_{i}$ is not the capacity of the node $V_{i}$ but a fraction of it, which means that voles are uncomfortable for $\Phi_{i}=R_{i}$ but they are not missing resources.

To introduce the initial boundary value problem at the node $V_{i}$ we consider the balance law 


$$
\partial_{t} \rho_{i}+\partial_{a} \rho_{i}=-\mathfrak{d}_{i}(t, a) \rho_{i}+P_{i}(t, a)
$$

where $t$ and $a$ are respectively the time and age variables, $\mathfrak{d}_{i}$ is the mortality rate at $V_{i}$, depending on time and age, and $P_{i}(t, a)$ is a source term which accounts for the possible departure/arrival of voles due to dispersal. The exact form of $P_{i}$ is given below.

Together with (2.4) we consider the initial condition

$$
\rho_{i}(0, a)=\rho_{0, i}(a)
$$

and the boundary condition

$$
\rho_{i}(t, 0)=\mathscr{A}_{i}\left(\Phi_{i}(t)\right) \int_{0}^{A_{3}} b_{i}(t, a) \rho_{i}(t, a) \mathrm{d} a,
$$

where $b_{i}$ is the reproduction rate of the node $V_{i}$ and the coefficient $\mathscr{A}_{i}\left(\Phi_{i}\right)$ reproduces Allee's effect, see [14]. This means that if the total population of a colony falls below a certain threshold, reproduction rate also diminishes and might become too small to compensate for mortality. The experimental observations suggest that this situation rarely occurs for voles, so that $\mathscr{A}_{i} \equiv 1$ is a reasonable choice in our simulations. However from the numerical point of view no additional difficulty comes from considering non constant $\mathscr{A}_{i}$, see also the Appendix A for a short additional discussion.

For $t>0$ given and $i \in\left\{1, \ldots, n_{\nu}\right\}$, we call $\mathscr{T}_{i}(t)=\left\{\tau_{1}^{i}, \ldots, \tau_{m_{i}(t)}^{i}\right\}$ the (possibly empty) set of times $\tau_{r}^{i}<t$ at which a dispersal outbreak took place from $V_{i}$. Then the term $P_{i}(t, a)$ in equation (2.4) takes the form

$$
\begin{aligned}
P_{i}(t, a) & =-\frac{c}{\eta} \chi_{\left[R_{i},+\infty\right)}\left(\sup _{z \in\left[(t-\eta)^{+}, t\right]} \Phi_{i}(z)\right) \chi_{\left[A_{1}, A_{2}\right)}(a) \rho_{i}(t, a) \\
& +\frac{c}{\eta} \sum_{\substack{j=1, \ldots, n_{v} \\
j \neq i}} \sum_{r=1}^{m_{j}(t)} \delta_{\tau_{r}^{j}}\left(t-\ell_{j i}\right) \chi_{\left[A_{1}, A_{2}\right)}\left(a-\ell_{j i}\right) K_{j i} \rho_{j}\left(t-\ell_{j i}, a-\ell_{j i}\right),
\end{aligned}
$$

where $\chi_{\left[R_{i},+\infty\right)}$ and $\chi_{\left[A_{1}, A_{2}\right)}$ are the characteristic functions of the intervals $\left[R_{i},+\infty\right)$ and $\left[A_{1}, A_{2}\right)$ respectively, $t \mapsto \delta_{\tau}(t)$ is the Kronecker delta centered at $\tau$ and we use the notation $x^{+}=\max (x, 0)$.

\subsection{A model with instantaneous dispersal (ID model)}

The model with gradual dispersal introduced above is very solid as it is based on classical PDE's for which a complete well-posedness theory is available. Moreover, the examples in the next section show that it reproduces the main features of the voles' population dynamics. Nevertheless, in order to bring the model to full size, on a graph counting several hundreds of nodes, one should overcome a number of technical difficulties in parallelization and, especially data storage. For this reason and in view of the possible construction of an hybrid PDE-Multi Agent model similar to the MLG, we propose here a second model which leads to a slightly less precise description of the dynamics, but should be easier to implement on large graphs.

Roughly speaking, we modify the previous model so that whenever the total population at a node $V_{i}$ reaches the threshold value $R_{i}$, the departure of dispersers takes place instantaneously. As a consequence, all the voles who left $V_{i}$ heading for $V_{j}$ will reach the node $V_{j}$ at the same time. These "instantaneous" departures/arrivals are easier to implement in a finite volume scheme and are not too demanding in term of data storage.

In order to write the model for the node $V_{i}$, we introduce $\mathscr{F}_{i}=\left\{\sigma_{0}^{i}, \sigma_{1}^{i}, \ldots, \sigma_{m_{i}}^{i}\right\}$, where $\sigma_{0}^{i}=0$ and $\sigma_{m_{i}}^{i}=T$, be the (possibly reduced to $\{0, T\}$ ) set of all the times between 0 and the final time $T$ at which, either a dispersal outbreak, or an arrival of voles, took place for the node $V_{i}$. 
Then between two times $\sigma_{K}^{i}$ and $\sigma_{K+1}^{i}$, where $K=0, \ldots, m_{i}-1$, the model can be written as follows

$$
\left\{\begin{aligned}
\partial_{t} \rho_{i}+\partial_{a} \rho_{i}=-\mathfrak{d}(t, a) \rho_{i}, \\
\rho_{i}(t, 0)=\mathscr{A}\left(\Phi_{i}(t)\right) \int_{0}^{A_{3}} b_{i}(t, a) \rho_{i}(t, a) \mathrm{d} a \\
\rho_{i}\left(\sigma_{K}^{i}+, a\right)=\chi_{\left[0, A_{1}\right) \cup\left[A_{2}, A_{3}\right]}(a) \rho_{i}\left(\sigma_{K}^{i}-, a\right)+\left(1-\chi_{\left[R_{i},+\infty\right)}\left(\Phi_{i}\left(\sigma_{K}^{i}-\right)\right)\right) \chi_{\left[A_{1}, A_{2}\right)}(a) \rho_{i}\left(\sigma_{K}^{i}-, a\right) \\
\quad+\sum_{\substack{j \neq i \\
j=1, \ldots, n_{v}}} \sum_{r=1}^{m_{j}-1} \delta_{\sigma_{r}^{j}}\left(\sigma_{K}^{i}-\ell_{j i}\right) \chi_{\left[R_{j},+\infty\right)}\left(\Phi_{j}\left(\sigma_{K}^{i}-\ell_{j i}\right)\right) \chi_{\left[A_{1}, A_{2}\right)}\left(a-\ell_{j i}\right) K_{j i} \rho_{j}\left(\sigma_{K}^{i}-\ell_{j i}, a-\ell_{j i}\right) .
\end{aligned}\right.
$$

\subsection{Reproduction and mortality rates}

Voles' reproduction season lasts typically from April to October, but variations are possible due to weather conditions and, of course, depending on the landscape characteristics. The ideal environment for fossorial voles is grassland where both food and shelter are easily available, but they can also settle in woodlands and farmlands. Mortality also depends on landscape characteristics as it is strongly related to the density and the kind of predators, but is everywhere higher during the winter season as food becomes scarce.

Based on [17, 11], the basic reproduction rate of a voles' population is 0.0165 females per female and per day during the reproduction season and only juvenile and adult individuals reproduce. In the time scale of our models this correspond to 0.3 per juvenile/adult vole per time unit. In Section 3.1, to validate our numerical schemes, we use as reproduction rate the piecewise constant function $b(a)=0.3 \chi_{\left[A_{1}, A_{3}\right](a)}$. Everywhere else in the paper we consider a smooth approximation of $b$, namely

$$
b_{1}(a)=\alpha \cdot \begin{cases}0.15 \exp \left(K\left(a-A_{1}-\varepsilon\right)\right) & \text { if } \quad 0 \leq a \leq A_{1}+\varepsilon \\ 0.3-0.15 \exp \left(-K\left(a-A_{1}-\varepsilon\right)\right) & \text { if } \quad a>A_{1}+\varepsilon\end{cases}
$$

where we set $K=10$ and $\varepsilon=0.2$. The parameter $\alpha \in(0,1]$ allows to adapt the average rate to specific situations, as we do in Sections 4.1 and 4.2.2.

Observations reported in [11] suggest that the average mortality rate during the reproduction season varies between 0.04 and 0.6 per 2 weeks, but the mortality rate for juvenile and newborn individuals is 1 to 3 times larger than for adults. Translated to our setting the value of mortality rate is between 0.05 and 0.77 per time unit. These informations lead us to define a mortality rate function of the following form

$$
\mathfrak{d}_{1}(a)=\delta \cdot \begin{cases}0.1-0.025 \exp \left(K\left(a-A_{2}-\varepsilon\right)\right) & \text { if } \quad 0 \leq a \leq A_{2}+\varepsilon, \\ 0.05+0.025 \exp \left(-K\left(a-A_{2}-\varepsilon\right)\right) & \text { if } \quad a>A_{2}+\varepsilon\end{cases}
$$

where $\delta>1$ is a parameter to be tuned.The graphs of $b_{1}$ and $\mathfrak{d}_{1}$ for $\alpha=\delta=1$ appear in Figure 1.

To validate our schemes it is enough to run simulations over a short interval of time, $t \in[0,1.3]$, which corresponds to less than one month in real time. In general however we wish to simulate the population dynamics over one or more years, so that we have to take into account the difference between the reproduction season, lasting from April to October, and the cold season where the reproduction stops and the mortality rate is more important, see for instance [8]. In our context, one year starting from April 1st corresponds to the period $[0, T]$, where $T=20$, and the reproduction season is $\left[0, T^{*}\right]$, where $T^{*}=12$. Given $\kappa>1$ we take

$$
K_{b}(t)=\left\{\begin{array}{lll}
1 & \text { if } & 0 \leq t \leq T^{*}, \\
0 & \text { if } & T^{*}<t \leq T,
\end{array} \quad \text { and } \quad K_{\mathfrak{d}}(t)=\left\{\begin{array}{llll}
1 & \text { if } & 0 \leq t \leq T^{*}, \\
\kappa & \text { if } & T^{*}<t \leq T,
\end{array}\right.\right.
$$




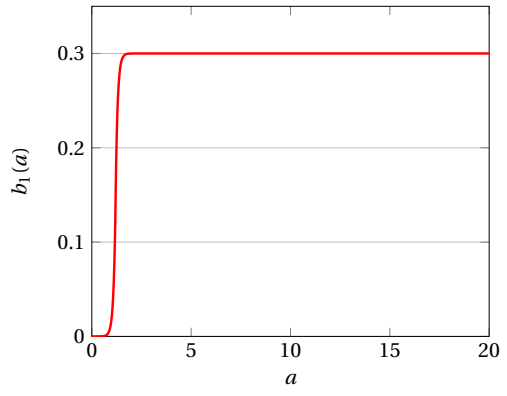

(a) The reproduction rate $b_{1}$ for $\alpha=1$.

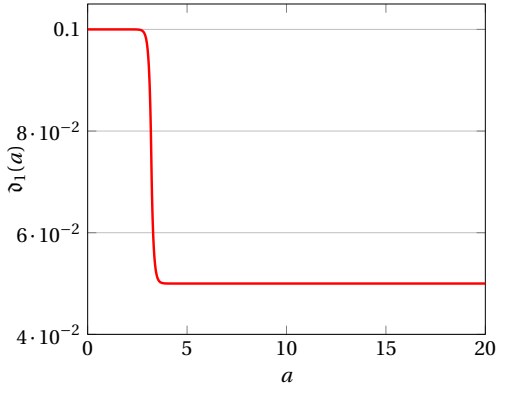

(b) The mortality rate $\mathfrak{d}_{1}$ for $\delta=1$.

Figure 1: The reproduction and mortality rates as functions of age.

and we compute the reproduction and mortality rates as functions of two variables defined on $[0, T] \times\left[0, A_{3}\right]$ as

$$
b_{K}(t, a)=K_{b}(t) b_{1}(a) \quad \text { and } \quad \mathfrak{d}_{K}(t, a)=K_{\mathfrak{d}}(t) \mathfrak{d}_{1}(a)
$$

The values of $\alpha, \delta$ and $\kappa$ will be specified for each of our examples.

To give a first, basic example of the evolution induced from the parameter functions above we consider the evolution internal to one single node over one year computed by the two models. The initial condition at $t=0$ consists of 70 adults equidistributed over their age class. We set $\alpha=1, \kappa=1.5$ and observe that if we choose $\delta=1$ several dispersals take place in both models starting at time $t \simeq 8.1$. After $T^{*}=12$, the total populations decreases and the only voles remaining at $t=T$ are the adult ones. Their total number is larger than the initial condition $(\simeq 90$ for the GD model and $\simeq 80$ for the ID model), so we say that the colony persists. On the other hand, if the mortality rate is higher, with $\delta=5.5$, the population declines, no dispersal occurs and the colony disappears before the end of the simulation.

The models coincide in this latter case, while in the first one we can observe several differences. Essentially, some of the juvenile voles do not leave the node during dispersal in the GD model, therefore the total population remains higher and we see more dispersals for the GD model than for the ID one.

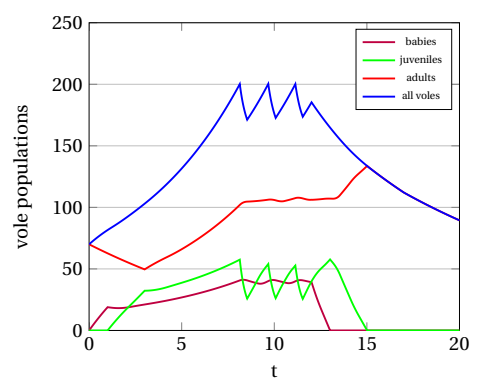

(a) GD model, persistence.

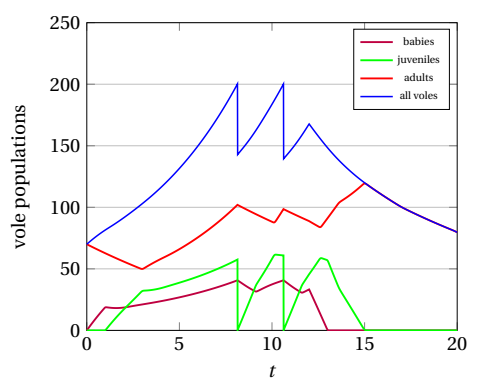

(b) ID model, persistence.

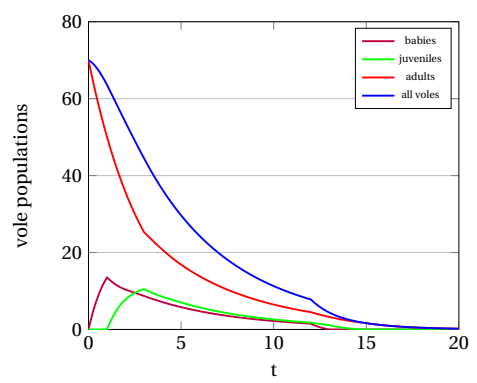

(c) Both models, extinction.

Figure 2: With reference to Section 2.3: the evolution of the vole population at one node. Left and center: low mortality in the GD and ID models respectively. Right : high mortality. 


\section{Finite volumes approximations of the models}

In this section, we propose finite volumes numerical schemes for the models described above and we validate them by comparison with exact solutions, for which analytical expressions exist and can be computed via the classical method of characteristics, [6, 16]. As the models rely on transport equations, the schemes are based on upwind schemes, [12].

Given a finite time horizon $T>0$ and a suitable maximal age $A_{3}$, we consider the computational domain [0,T] $\times$ $\left[0, A_{3}\right]$ and let $\Delta t$ and $\Delta a$ be respectively the constant time and age steps. We set $N_{T}=\lfloor T / \Delta t\rfloor, N_{1}=\left\lfloor A_{1} / \Delta a\right\rfloor, N_{2}=$ $\left\lfloor A_{2} / \Delta a\right\rfloor$ and $N_{A}=\left\lfloor A_{3} / \Delta a\right\rfloor$, where $\lfloor s\rfloor$ stands for the integer part of $s$. Then for any $1 \leq h \leq N_{A}$, we introduce the cells $K_{h}=\left[a_{h-1 / 2}, a_{h+1 / 2}\right)$, the cells centers $a_{h}=(h-1 / 2) \Delta a$ and, for $0 \leq n \leq N_{T}$, we define the times $t^{n}=n \Delta t$. For any given $i, j \in\left\{1, \ldots, n_{v}\right\}, i \neq j$, we approximate the edge $\left(V_{i}, V_{j}, \ell_{i j}\right)$ by $\left(V_{i}, V_{j}, L_{i j} \Delta t\right)$, where $L_{i j}=\left\lfloor\ell_{i j} / \Delta t\right\rfloor$. Analogously, we approximate the age increase during dispersal by $L_{i j}^{*} \Delta a$ where $L_{i j}^{*}=\left\lfloor\ell_{i j} / \Delta a\right\rfloor$.

Given a node $V_{i}, 1 \leq i \leq n_{v}$, we denote by $b_{i, h}^{n}, \mathfrak{d}_{i, h}^{n}$ and $\rho_{i, h}^{n}$, for $1 \leq h \leq N_{A}$ and $0 \leq n \leq N_{T}$, the approximation of the averages of $b_{i}\left(t^{n}, \cdot\right), \mathfrak{d}_{i}\left(t^{n}, \cdot\right)$ and $\rho_{i}\left(t^{n}, \cdot\right)$ on the cell $K_{h}$, namely

$$
\begin{array}{lll}
b_{i, h}^{n}=\frac{1}{\Delta a} \int_{K_{h}} b_{i}\left(t^{n}, a\right) \mathrm{d} a & , & \mathfrak{d}_{i, h}^{n}=\frac{1}{\Delta a} \int_{K_{h}} \mathfrak{d}_{i}\left(t^{n}, a\right) \mathrm{d} a, \\
\rho_{i, h}^{0}=\frac{1}{\Delta a} \int_{K_{h}} \rho_{i, 0}(a) \mathrm{d} a & \text { and } & \rho_{i, h}^{n} \simeq \frac{1}{\Delta a} \int_{K_{h}} \rho\left(t^{n}, a\right) \mathrm{d} a \text { if } \quad n>0 .
\end{array}
$$

For each $i \in\left\{1, \ldots, n_{v}\right\}$ and $n \in\left\{0, \ldots, N_{T}\right\}$ the total population of voles in $V_{i}$ at time $t^{n}$ is

$$
\Phi_{i}^{n}=\Delta a \sum_{h=1}^{N_{A}} \rho_{i, h}^{n}
$$

The numerical scheme for the GD model For any given value of the parameter $\eta>0$, minimal time span of any dispersal in our model, we introduce the number of time steps $N_{\eta}=\lfloor\eta / \Delta t\rfloor$ and we fix to $N_{\eta} \Delta t$ the minimal duration of a dispersal in the numerical scheme. For $n \in \mathbb{N}$ fixed and $\tau_{K}^{i}$ in $\mathscr{T}_{i}\left(t^{n}\right)=\left\{\tau_{1}^{i}, \ldots, \tau_{m_{i}\left(t^{n}\right)}^{i}\right\}$, the set of outbreak times taking place from $V_{i}$ before $t^{n}$, we introduce $N_{\tau_{K}^{i}}=\left\lfloor\tau_{K}^{i} / \Delta t\right\rfloor$. We next define

$$
\widetilde{\Phi}_{i}^{n}=\sup _{\left(n-N_{\eta}\right)^{+} \leq m \leq n} \Phi_{i}^{m} \quad, \quad N_{K}^{i j}=N_{\tau_{K}^{i}}+L_{i j} \quad \text { and } \quad \delta_{N_{K}^{i j}}^{n}= \begin{cases}1 & \text { if } n=N_{K}^{i j} \\ 0 & \text { otherwise. }\end{cases}
$$

We finally set

$$
d_{i, h}^{n+1}=1+\Delta t\left(\mathfrak{d}_{i, h}^{n+1}+\frac{c}{\eta} \chi_{\left[R_{i},+\infty\right)}\left(\widetilde{\Phi}_{i}^{n}\right) \chi_{\left[N_{1}, N_{1}+N_{2}\right)}(h)\right)
$$

which comes from the implicit treatment of the mortality term in (2.4) and the semi-implicit treatment of the dispersal term in (2.7). Then, writing a standard upwind scheme for the transport part of (2.4) and using an explicit treatment of the last term of (2.7) we obtain the following scheme

$$
\left\{\begin{array}{l}
\rho_{i, h}^{n+1} \quad=\frac{1}{d_{i, h}^{n+1}}\left[\left(1-\frac{\Delta t}{\Delta a}\right) \rho_{i, h}^{n}+\frac{\Delta t}{\Delta a} \rho_{i, h-1}^{n}+c \frac{\Delta t}{\eta} \sum_{\substack{j \neq i \\
j=1, \ldots, n_{v}}} \sum_{K=1}^{m_{j}\left(t^{n}\right)} \delta_{N_{K}^{j i}}^{n} \chi_{\left[N_{1}, N_{1}+N_{2}\right]}\left(h-L_{j i}^{*}\right) K_{j i} \rho_{j, h-L_{j i}^{*}}^{n-L_{j i}}\right] \\
\quad \text { for } 1 \leq i \leq n_{v}, 1 \leq h \leq N_{A}, 0 \leq n \leq N_{T}-1, \\
\rho_{i, 0}^{n} \quad=\mathscr{A}_{i}\left(\Phi_{i}^{n}\right) \Delta a \sum_{h=1}^{N_{A}} b_{i, h}^{n} \rho_{i, h}^{n}, \quad 1 \leq i \leq n_{\nu}, 0 \leq n \leq N_{T}-1 .
\end{array}\right.
$$


The numerical scheme for the ID model For $n \in \mathbb{N}$, let $\sigma_{K}^{i}$ be in the set $\mathscr{F}_{i}=\left\{\sigma_{1}^{i}, \ldots, \sigma_{m_{i}}^{i}\right\}$ of times at which either a dispersal or an arrival took place in the node $V_{i}$ before $t^{n}$. We introduce $N_{\sigma_{K}^{i}}=\left\lfloor\sigma_{K}^{i} / \Delta t\right\rfloor$.

Writing again a standard upwind scheme for (2.8), we get for each $i \in\left\{1, \ldots, n_{v}\right\}$,

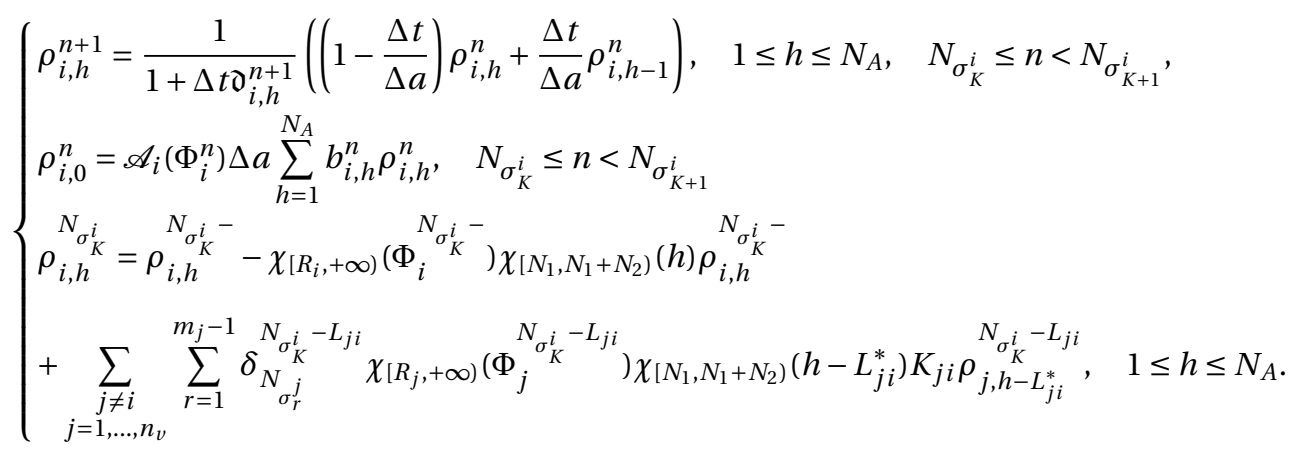

\subsection{Validation}

In this part, we validate the implementation of the numerical schemes described above by comparison to exact solutions for which an analytical expression exists.

We consider a simple graph consisting of two nodes $V_{1}$ and $V_{2}$ and, denoting by $\mathscr{L}$ the matrix of the travel times and $\mathbf{K}$ the distribution matrix, we set

$$
\mathscr{L}=\left(\begin{array}{cc}
0 & 0.5 \\
0.5 & 0
\end{array}\right) \quad \text { and } \quad \mathbf{K}=\left(\begin{array}{ll}
0 & 1 \\
1 & 0
\end{array}\right)
$$

which means that, during a dispersal in the node $V_{1}$, all the juvenile voles will move to the node $V_{2}$ and vice versa. The initial conditions at the nodes are the following

- At $V_{1}$, we consider 50 juveniles and 170 adults. Individuals are equidistributed over their age class.

$$
\rho_{1}(0, a)=\frac{50}{A_{2}-A_{1}} \chi_{\left[A_{1}, A_{2}\right)}(a)+\frac{170}{A_{3}-A_{2}} \chi_{\left[A_{2}, A_{3}\right]}(a) .
$$

- At $V_{2}$, we consider 120 adults. Individuals are equidistributed over this age class.

$$
\rho_{2}(0, a)=\frac{120}{A_{3}-A_{2}} \chi_{\left[A_{2}, A_{3}\right]}(a) .
$$

For simplicity we assume all the other parameters of our models to be piecewise constant and identical on the two nodes

- Reproduction rate: $b(a)=b \chi_{\left[A_{1}, A_{3}\right]}(a)=0.3 \chi_{\left[A_{1}, A_{3}\right]}(a)$;

- Mortality rate: $\mathfrak{d}(a)=\mathfrak{d}_{b j} \chi_{\left[0, A_{2}\right)}(a)+\mathfrak{d}_{a d} \chi_{\left[A_{2}, A_{3}\right]}(a)=0.2 \chi_{\left[0, A_{2}\right)}(a)+0.1 \chi_{\left[A_{2}, A_{3}\right]}(a)$;

- Dispersal threshold: $R=200$;

- Mortality rate during dispersal: $\mathfrak{d}_{d i s}(a)=0.5$.

For the GD model we set $c=1$ and we also have to fix the value of the minimal time span of dispersal and we set $\eta=0.25$. 


\subsubsection{The exact solution for the GD model}

In this section we provide a detailed description of the explicit solution in the time interval [0,1.3] for the GD model with the settings and initial conditions introduced in Section 3.1. The travel time between the two nodes is $\ell=0.5$.

Solution at $V_{1}$ during the first dispersal, $t \in\left[0, t_{1}\right]$. The given initial condition makes the total population in $V_{1}$ at $t=0$ larger than the dispersal threshold $R$ and triggers a first dispersal. The density of voles in $V_{1}$ is a function of time and age, defined piecewise with respect to age

$$
\rho_{1}(t, a)= \begin{cases}\operatorname{babies}_{1}(t, a)=b e^{-\mathfrak{d}_{b j} a} \int_{A_{1}}^{A_{3}} \rho_{1}(t-a, c) \mathrm{d} c, & a \in[0, t), \\ 0, & a \in\left[t, A_{1}+t\right), \\ \operatorname{juv}_{1}(t, a)=\frac{50}{A_{2}-A_{1}} e^{-\left(\mathfrak{d}_{b j}+1 / \eta\right) t}=25 e^{-4.2 t}, & a \in\left[A_{1}+t, A_{2}\right), \\ \operatorname{newad}_{1}(t, a)=\frac{50}{A_{2}-A_{1}} e^{-\left(\mathfrak{d}_{b j}+1 / \eta\right)\left(t-\left(a-A_{2}\right)\right)-\mathfrak{d}_{a d}\left(a-A_{2}\right)}=25 e^{-4.2\left(t-\left(a-A_{2}\right)\right)-0.1\left(a-A_{2}\right)}, & a \in\left[A_{2}, A_{2}+t\right), \\ \operatorname{adults}_{1}(t, a)=\frac{170}{A_{3}-A_{2}} e^{-\mathfrak{d}_{a d} t}=10 e^{-0.1 t}, & a \in\left[A_{2}+t, A_{3}\right] .\end{cases}
$$

We observe that the total density of juvenile voles of age $a$ who have left $V_{1}$ at time $t$ is given by

$$
\frac{50}{A_{2}-A_{1}} e^{-\mathfrak{d}_{b j} t}-\mathrm{juv}_{1}(t, a),
$$

so that the density of voles of age $a$ leaving $V_{1}$ at time $t$ is

$$
\operatorname{disp}(t, a)=\frac{50}{A_{2}-A_{1}} e^{-\mathfrak{d}_{b j} t}\left(-\mathfrak{d}_{b j}+\left(\mathfrak{d}_{b j}+\frac{1}{\eta}\right) e^{-t / \eta}\right) .
$$

The value of the total population in $V_{1}, \Phi_{1}$, equals the threshold $R$ at $t_{0} \simeq 0.18755$. From this moment the dispersal continues for a time $\eta$ more, and we denote by $t_{1}=t_{0}+\eta \simeq 0.43755$ the instant at which it ends.

Solution at $V_{1}$ between two dispersals, $t \in\left[t_{1}, t_{2}\right]$. The function $\rho_{1}$ is still piecewise defined with respect to the age variable, but its components are different. The evolution of the component juv is juv $_{12}$. The new components correspond to the babies born at $t>t_{1}$, babies $_{2}$, and to the voles passing from juveniles to adults at $t>t_{1}$, newad ${ }_{2}$.

$$
\rho_{1}(t, a)= \begin{cases}\operatorname{babies}_{2}(t, a)=b e^{-\mathfrak{d}_{b j} a} \int_{A_{1}}^{A_{3}} \rho_{1}(t-a, c) \mathrm{d} c, & a \in\left[0, t-t_{1}\right), \\ \operatorname{juv}_{12}(t, a)=\operatorname{juv}_{1}\left(t_{1}, a-\left(t-t_{1}\right)\right) e^{-\mathfrak{d}_{b j}\left(t-t_{1}\right)}, & a \in\left[A_{1}+t, A_{2}\right), \\ \operatorname{newad}_{2}(t, a)=\operatorname{juv}_{1}\left(t_{1}, a-\left(t-t_{1}\right)\right) e^{-\mathfrak{d}_{b j}\left(t-t_{1}-\left(a-A_{2}\right)\right)-\mathfrak{d}_{a d}\left(a-A_{2}\right)}, & a \in\left[A_{2}, A_{2}+\left(t-t_{1}\right)\right), \\ \rho_{1}\left(t_{1}, a-\left(t-t_{1}\right)\right) e^{-\mathfrak{d}(a)\left(t-t_{1}\right)}, & \text { otherwise. }\end{cases}
$$

The total population at $V_{1}, \Phi_{1}$, increases up to reach again the value $R$ at $t=t_{2} \simeq 0.84818$. This triggers a second dispersal.

Solution at $V_{1}$ for $t \in\left[t_{2}, A_{1}\right]$. The second dispersal modifies the dynamics of the juveniles, so that the functions giving the density of newborns and the density of new adults also need to be updated. The evolution of the component juv $_{12}$ is juv jus $_{12}$.

$$
\rho_{1}(t, a)= \begin{cases}\operatorname{babies}_{3}(t, a)=b e^{-\mathfrak{d}_{b j} a} \int_{A_{1}}^{A_{3}} \rho_{1}(t-a, c) \mathrm{d} c, & a \in\left[0, t-t_{2}\right), \\ \operatorname{juv}_{123}(t, a)=\operatorname{juv}_{12}\left(t_{2}, a-\left(t-t_{2}\right)\right) e^{-\left(\mathfrak{d}_{b j}+1 / \eta\right)\left(t-t_{2}\right)}, & a \in\left[A_{1}+t, A_{2}\right), \\ \operatorname{newad}_{3}(t, a)=\operatorname{juv}_{12}\left(t_{2}, a-\left(t-t_{2}\right)\right) e^{-\left(\mathfrak{d}_{b j}+1 / \eta\right)\left(t-t_{2}-\left(a-A_{2}\right)\right)-\mathfrak{d}_{a d}\left(a-A_{2}\right)}, & a \in\left[A_{2}, A_{2}+\left(t-t_{2}\right)\right), \\ \rho_{1}\left(t_{2}, a-\left(t-t_{2}\right)\right) e^{-\mathfrak{d}(a)\left(t-t_{2}\right)} & \text { otherwise. }\end{cases}
$$


Observe the total population in $V_{1}$ reaches again the value $R$ at time $t_{3} \simeq 0.94675$. This does not produce an immediate change in the dynamics because the difference $t_{3}-t_{2}$ is less that $\eta$, but tells us that the second dispersal will last up to time $t_{4}=t_{3}+\eta \simeq 1.44675$.

Solution at $V_{1}$ for $t \in\left[A_{1}, 1.3\right]$. For $t>A_{1}$ we have to consider two additional age classes : some of the babies becomes juvenile and start to reproduce, we denote them by newjuv , $_{\text {, and babies born after time } t=A_{1} \text {, babies }}$.

$$
\rho_{1}(t, a)= \begin{cases}\operatorname{babies}_{4}(t, a)=b e^{-\mathfrak{d}_{b j} a} \int_{A_{1}}^{A_{3}} \rho_{1}(t-a, c) \mathrm{d} c, & a \in\left[0, t-A_{1}\right), \\ \operatorname{babies}_{1}(t, a), & a \in\left[t-t_{1}, A_{1}\right), \\ \operatorname{newjuv}_{4}(t, a)=\operatorname{babies}_{1}\left(A_{1}, a-\left(t-A_{1}\right)\right) e^{-\mathfrak{d}_{b j}(t-a)-\left(\mathfrak{d}_{b j}+1 / \eta\right)\left(a-A_{1}\right)} & a \in\left[A_{1}, t\right), \\ \rho_{1}\left(A_{1}, a-\left(t-A_{1}\right)\right) e^{-\mathfrak{d}(a)\left(t-A_{1}\right)}, & \text { otherwise. }\end{cases}
$$

Solution at $V_{2}$ before the arrival of voles from $V_{1}, t \in[0, \ell]$. At time $t=0$ the total population at $V_{2}$ is 120 . We will see that no dispersal takes place from this node for $t=[0,1.3]$. We use a different font to distinguish the age groups appearing in the solution at node $V_{2}$, while the indices are given in the same way.

$$
\rho_{2}(t, a)= \begin{cases}\operatorname{babies}_{1}(t, a)=b e^{-\mathfrak{d}_{b j} a} \int_{A_{1}}^{A_{3}} \rho_{2}(t-a, c) \mathrm{d} c, & a \in[0, t), \\ 0 & a \in\left[t, A_{2}+t\right), \\ \operatorname{adults}_{1}(t, a)=\frac{120}{A_{3}-A_{2}} e^{-\mathfrak{d}_{a d} t}=\frac{120}{17} e^{-0.1 t}, & a \in\left[A_{2}+t, A_{3}\right] .\end{cases}
$$

Solution at $V_{2}$ during the arrival of the first group of dispersers, $t \in\left[\ell, \ell+t_{1}\right]$. We have to take into account several new groups of individuals. The new components correspond to the babies born at $t>\ell$, babies 2 , and to the voles arriving from the node $V_{1}$. Some of them arrive as juveniles, juv $v_{2}$, some as adults, adults $s_{2}$. Some of the juveniles become adults after their arrival, we call them newad 2 . The voles whose age is in $\left(A_{2}+\ell, A_{2}+t\right)$ originated from $V_{1}$, but when they arrived they were jounger than $A_{2}+\ell$, we call this group adultsevol $l_{2}$. We compute their density using (3.20) and the fact that while travelling from $V_{1}$ to $V_{2}$ the dispersers solve the ODE $y^{\prime}=-\mathfrak{d}_{\text {dis }} y$.

$$
\rho_{2}(t, a)= \begin{cases}\operatorname{babies}_{2}(t, a)=b e^{-\mathfrak{d}_{b j} a} \int_{A_{1}}^{A_{3}} \rho_{2}(t-a, c) \mathrm{d} c, & a \in[0, t-\ell), \\ \operatorname{juv}_{2}(t, a)=\int_{\ell}^{t} e^{-\mathfrak{d}_{b j}(t-s)} e^{-\mathfrak{d}_{d i s} \ell} \operatorname{disp}(s-\ell, a-(t-s+\ell)) \mathrm{d} s, & a \in\left[A_{1}+t, A_{2}\right), \\ \operatorname{newad}_{2}(t, a)+\operatorname{adults}_{2}(t, a)=\operatorname{juv}_{2}\left(t-\left(a-A_{2}\right), A_{2}\right) e^{-\mathfrak{d}_{a d}\left(a-A_{2}\right)} & \\ +\int_{t-\left(a-A_{2}\right)}^{t} e^{-\mathfrak{d}_{a d}(t-s)} e^{-\mathfrak{d}_{d i s} \ell} \operatorname{disp}(s-\ell, a-(t-s+\ell)) \mathrm{d} s, & a \in\left[A_{2}, A_{2}+(t-\ell)\right), \\ \operatorname{adults}_{2}(t, a)=\int_{\ell}^{t} e^{-\mathfrak{d}_{a d}(t-s)} e^{-\mathfrak{d}_{d i s} \ell} \operatorname{disp}(s-\ell, a-(t-s+\ell)) \mathrm{d} s, & a \in\left[A_{2}+(t-\ell), A_{2}+\ell\right), \\ \operatorname{adultsevol}_{2}(t, a)=\operatorname{adults}_{2}\left(t-\left(a-\left(A_{2}+\ell\right)\right), A_{2}+\ell\right) e^{-\mathfrak{d}_{a d}\left(a-\left(A_{2}+\ell\right)\right)}, & a \in\left[A_{2}+\ell, A_{2}+t\right), \\ \rho_{2}(\ell, a-(t-\ell)) e^{-\mathfrak{d}(a)(t-\ell)}, & \text { otherwise. }\end{cases}
$$

Solution at $V_{2}$ for $t \in\left[\ell+t_{1}, 1.3\right]$. In the interval of time $\left[\ell+t_{1}, \ell+t_{2}\right]$ no more voles arrive from $V_{1}$ and the total population of $V_{2}$ does not attain the threshold value $R$. For our validation we only compute the solution up to time $t=1.3<\ell+t_{2}$. The age groups already present in the dynamics just evolve following the prescribed mortality rates,

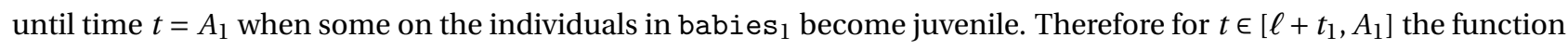


$\rho_{2}$ takes the form

$$
\rho_{2}(t, a)= \begin{cases}\operatorname{babies}_{3}(t, a)=b e^{-\mathfrak{d}_{b j} a} \int_{A_{1}}^{A_{3}} \rho_{2}(t-a, c) \mathrm{d} c, & a \in\left[0, t-\left(\ell+t_{1}\right)\right), \\ \operatorname{newad}_{3}(t, a)=\operatorname{juv}_{2}\left(\ell+t_{1}, a-\left(t-\ell-t_{1}\right)\right) e^{-\mathfrak{d}_{b j}\left(t-\left(\ell+t_{1}\right)-\left(a-A_{2}\right)\right)-\mathfrak{d}_{a d}\left(a-A_{2}\right)}, & a \in\left[A_{2}, A_{2}+t-\left(\ell+t_{1}\right)\right), \\ \rho_{2}\left(\ell+t_{1}, a-\left(t-\ell-t_{1}\right)\right) e^{-\mathfrak{d}(a)\left(t-\left(\ell+t_{1}\right)\right),} & \text { otherwise. }\end{cases}
$$

While for $t \in\left[A_{1}, 1.3\right] \rho_{2}$ becomes

$$
\rho_{2}(t, a)= \begin{cases}\operatorname{babies}_{4}(t, a)=b e^{-\mathfrak{d}_{b j} a} \int_{A_{1}}^{A_{3}} \rho_{2}(t-a, c) \mathrm{d} c, & a \in\left[0, t-A_{1}\right), \\ \operatorname{babies}_{1}(t, a), & a \in\left[t-A_{1}, A_{1}\right), \\ \operatorname{newjuv}_{4}(t, a)=\operatorname{babies}_{1}\left(A_{1}, a-\left(t-A_{1}\right)\right) e^{-\mathfrak{d}_{b j}\left(t-A_{1}\right)}, & a \in\left[A_{1}, t\right), \\ \operatorname{newad}_{4}(t, a)=\operatorname{juv}_{2}\left(A_{1}, a-\left(t-A_{1}\right)\right) e^{-\mathfrak{d}_{b j}\left(t-A_{1}-\left(a-A_{2}\right)\right)-\mathfrak{d}_{a d}\left(a-A_{2}\right)}, & a \in\left[A_{2}, A_{2}+t-A_{1}\right), \\ \rho_{2}\left(A_{1}, a-\left(t-A_{1}\right)\right) e^{-\mathfrak{d}(a)\left(t-A_{1}\right)}, & \text { otherwise. }\end{cases}
$$

Figure 3 shows the comparison between the exact and the numerical solutions at the two nodes for the GD model at the final time $T=1.3$. We observe a perfect matching between the two solutions where the numerical one was computed with $\Delta a=\Delta t=0.00625$.

\subsubsection{The exact solution for the ID model}

In this section we provide a detailed description of the explicit solution in the time interval $[0,1.3]$ for the ID model with the settings and initial conditions introduced in Section 3.1. The travel time between the two nodes is $\ell=0.5$.

Solution at $V_{1}$ for $t \in\left[0, A_{1}\right]$. The given initial condition makes the total population in $V_{1}$ at $t=0$ higher than the dispersal threshold $R$, therefore the juvenile individuals immediately leave the node. The density of voles in $V_{1}$ is a function of time and age, defined piecewise with respect to age

$$
\rho_{1}(t, a)= \begin{cases}\operatorname{babies}_{1}(t, a)=b e^{-\mathfrak{d}_{b j} a} \int_{A_{1}}^{A_{3}} \rho_{1}(t-a, c) \mathrm{d} c, & a \in[0, t) \\ 0, & a \in\left[t, A_{2}+t\right), \\ \operatorname{adults}_{1}(t, a)=\frac{170}{A_{3}-A_{2}} e^{-\mathfrak{d}_{a d} t}=10 e^{-0.1 t}, & a \in\left[A_{2}+t, A_{3}\right] .\end{cases}
$$

Solution at $V_{1}$ for $t \in\left[A_{1}, 1.3\right]$. Starting from time $t=A_{1}$ a fraction of the babies will become juveniles and their reproductive process has to be taken into account. This leads to the formation of two more age groups: the babies born after time $t=A_{1}$, babies $_{2}$, and the individuals passing from the babies to juveniles, newjuv 2 .

$$
\rho_{1}(t, a)= \begin{cases}\operatorname{babies}_{2}(t, a)=b e^{-\mathfrak{d}_{b j} a} \int_{A_{1}}^{A_{3}} \rho_{1}(t-a, c) \mathrm{d} c, & a \in\left[0, t-A_{1}\right), \\ \operatorname{babies}_{1}(t, a), & a \in\left[t-A_{1}, A_{1}\right), \\ \operatorname{newjuv}_{2}(t, a)=\operatorname{babies}_{1}\left(A_{1}, a-\left(t-A_{1}\right)\right) e^{-\mathfrak{d}_{b j}\left(t-A_{1}\right)}, & a \in\left[A_{1}, t\right), \\ 0, & a \in\left[t, A_{2}+t\right), \\ \operatorname{adults}_{1}(t, a)=\operatorname{adults}_{1}\left(A_{1}, a-\left(t-A_{1}\right)\right) e^{-\mathfrak{d}_{a d}\left(t-A_{1}\right)}, & a \in\left[A_{2}+t, A_{3}\right] .\end{cases}
$$

Solution at $V_{2}$ before the arrival of voles from $V_{1}, t \in[0, \ell]$. The dynamics is the same in the two models. The explicit form of the solution is given in (3.24). 


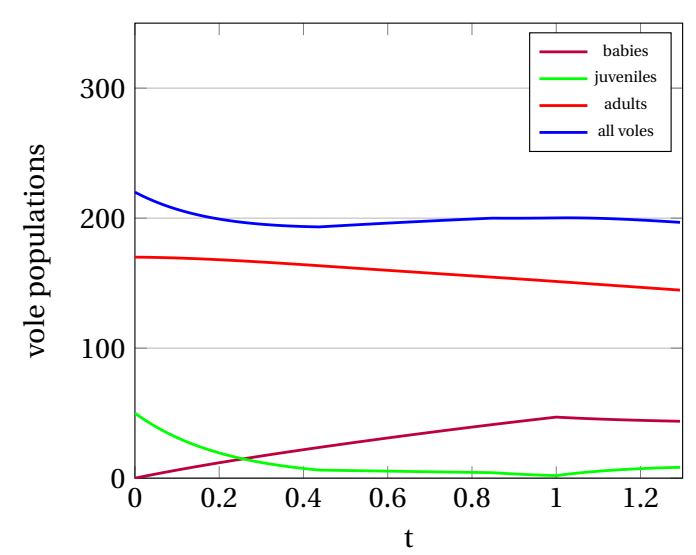

(a) Exact solution for GD model for node $V_{1}$

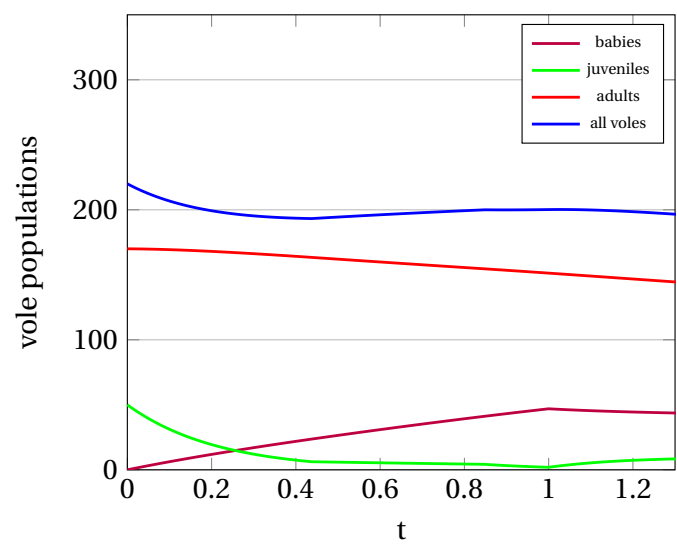

(c) Computed solution for GD model for node $V_{1}$

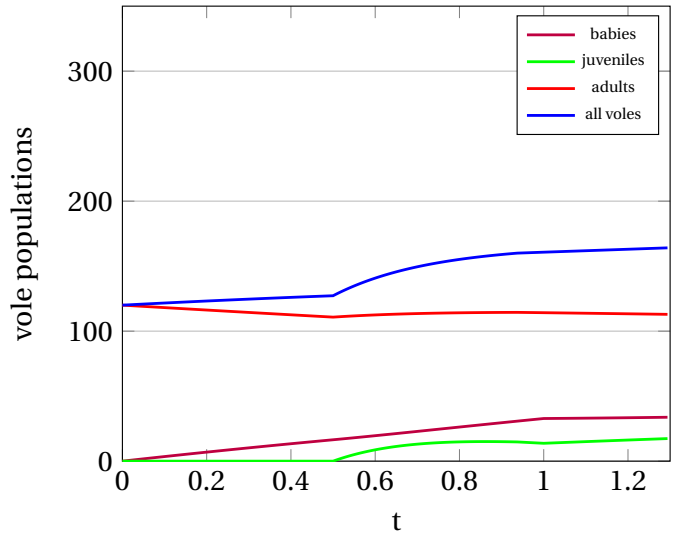

(b) Exact solution for GD model for node $V_{2}$

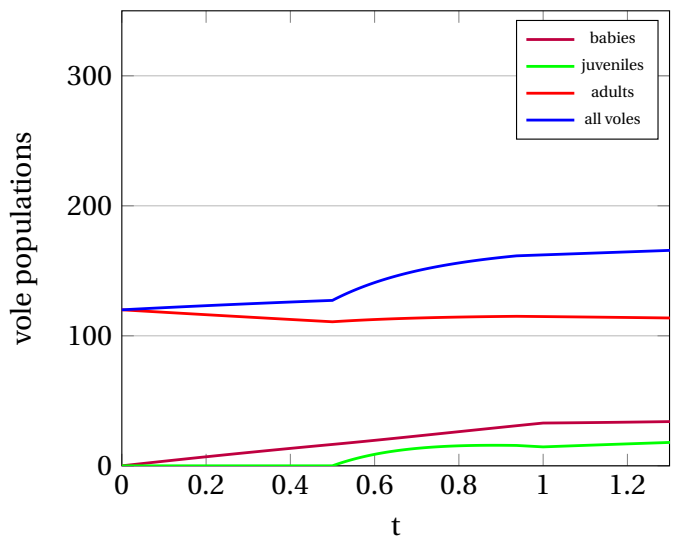

(d) Computed solution for GD model for node $V_{2}$

Figure 3: With reference to Section 3.1.1: the comparison between the population of voles in the exact solution and the GD model for $\eta=0.25$.

Solution at $V_{2}$ for $t \in\left[\ell, A_{1}\right]$. The density of voles reaching $V_{2}$ at $t=\ell=0.5$ are $\frac{50}{A_{2}-A_{1}} \chi_{\left[A_{1}+\ell, A_{2}+\ell\right]}(a) e^{-\ell \mathfrak{d}_{d i s}}$, then some of them are juveniles, juv $v_{2}$, some are adults, adults $s_{2}$. Some of the juveniles become adults after their arrival, we call them newad . We call babies $_{2}$, the babies born for $t>\ell$.

$$
\rho_{2}(t, a)= \begin{cases}\operatorname{babies}_{2}(t, a)=b e^{-\mathfrak{d}_{b j} a} \int_{A_{1}}^{A_{3}} \rho_{2}(t-a, c) \mathrm{d} c, & a \in[0, t-\ell), \\ \operatorname{juv}_{2}(t, a)=\frac{50}{A_{2}-A_{1}} e^{-\ell \mathfrak{d}_{d i s}-\mathfrak{d}_{b j}(t-\ell)}, & a \in\left[A_{1}+t, A_{2}\right), \\ \operatorname{newad}_{2}(t, a)=\operatorname{juv}_{2}\left(t-\left(a-A_{2}\right), A_{2}\right) e^{-\mathfrak{d}_{a d}\left(a-A_{2}\right)}, & a \in\left[A_{2}, A_{2}+(t-\ell)\right), \\ \operatorname{adults}_{2}(t, a)=\frac{50}{A_{2}-A_{1}} e^{-\ell \mathfrak{d}_{d i s}-\mathfrak{d}_{a d}(t-\ell)}, & a \in\left[A_{2}+(t-\ell), A_{2}+t\right), \\ \rho_{2}(\ell, a-(t-\ell)) e^{-\mathfrak{d}(a)(t-\ell)}, & \text { otherwise. }\end{cases}
$$

Solution at $V_{2}$ for $t \in\left[A_{1}, 1.3\right]$. Starting from time $t=A_{1}$ a fraction of the babies will become juveniles and their reproductive process has to be taken into account. This leads to the formation of two more age groups : the babies born after time $t=A_{1}$, babies 3 , and the individuals passing from the babies to juveniles, newjuv . $_{3}$ 


$$
\rho_{2}(t, a)= \begin{cases}\operatorname{babies}_{3}(t, a)=b e^{-\mathfrak{d}_{b j} a} \int_{A_{1}}^{A_{3}} \rho_{2}(t-a, c) d c, & a \in\left[0, t-A_{1}\right), \\ \operatorname{babies}_{1}(t, a), & a \in\left[t-\ell, A_{1}\right), \\ \operatorname{newjuv}_{3}(t, a)=\operatorname{babies}_{1}\left(A_{1}, a-\left(t-A_{1}\right)\right) e^{-\mathfrak{d}_{b j}\left(t-A_{1}\right)}, & a \in\left[A_{1}, t\right), \\ \rho_{2}\left(A_{1}, a-\left(t-A_{1}\right)\right) e^{-\mathfrak{d}(a)\left(t-A_{1}\right)}, & \text { otherwise. }\end{cases}
$$

Figure 4 illustrates the comparison between the explicit solution and its numerical solution at the two nodes for the ID model at the final time $T=1.3$. We again observe a perfect agreement between the two solutions. The numerical solutions was computed with $\Delta a=\Delta t=0.00625$.

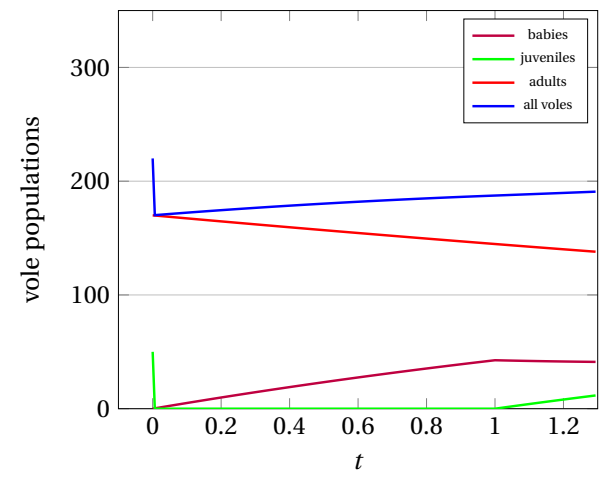

(a) Node $V_{1}$

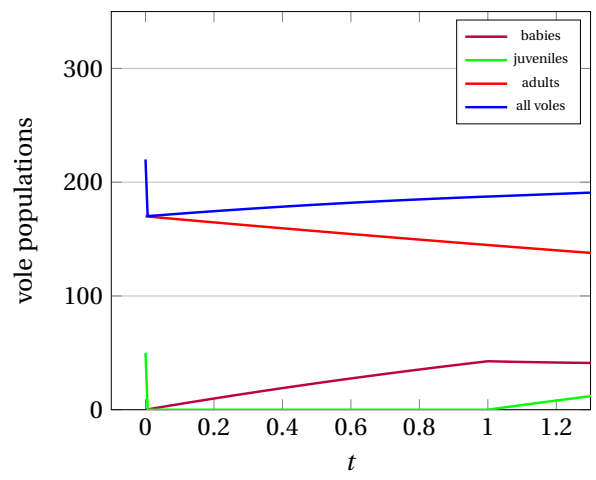

(c) Node $V_{1}$

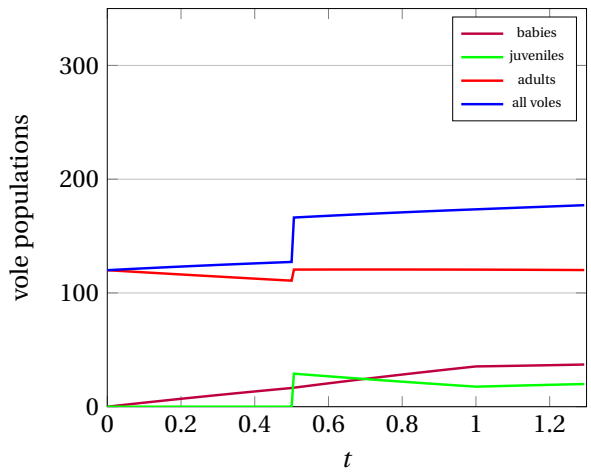

(b) Node $V_{2}$

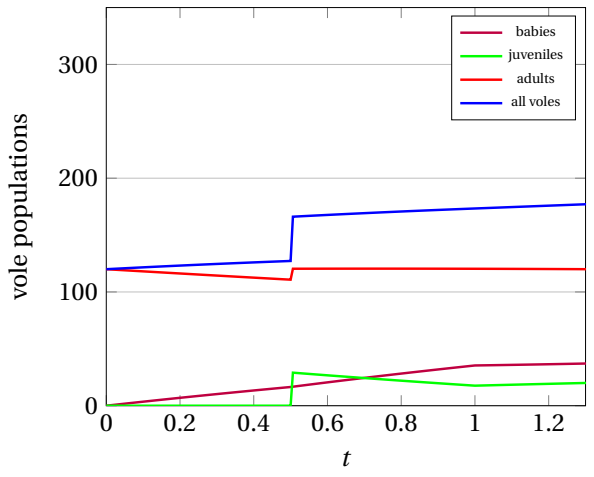

(d) Node $V_{2}$

Figure 4: With reference to Section 3.1.1: the comparison between the population of voles in the exact solution and in the ID model.

\subsection{Numerical convergence}

We introduce here the following relative $L^{1}$-discrete error for the density in the node $V_{i}, i=1,2$, at a given time $t^{n}$

$$
e_{i}^{n}=\frac{\sum_{1 \leq h \leq N_{a}}\left|\rho_{i}\left(t^{n}, a_{h}\right)-\rho_{i, h}^{n}\right|}{\sum_{1 \leq h \leq N_{a}}\left|\rho_{i}\left(t^{n}, a_{h}\right)\right|},
$$

where $\rho\left(t^{n}, a_{h}\right)$ is an exact solution evaluated at the point $a_{h}$ and at time $t^{n}$. 
In tables 1 and 2 , we have reported the relative $L^{1}$-discrete errors for both models in the two nodes $V_{1}$ and $V_{2}$, computed for different values of age and times steps at two times $T=0.6$ and $T=1.2$. The results suggest the convergence of the numerical schemes. Moreover, figures 5 and 6 suggest that the order of convergence of the scheme for the GD model is between 0.71 and 1 , and the one for the ID model is 1 . We can also observe that the loss of accuracy of the scheme for the GD model is mainly due to the approximation of the arrival term in (2.7).

\begin{tabular}{|c|c|c|c|c|c|}
\hline$\Delta t$ & $\Delta a$ & $e_{1}^{N_{T}}, T=0.6$ & $e_{2}^{N_{T}}, T=0.6$ & $e_{1}^{N_{T}}, T=1.2$ & $e_{2}^{N_{T}}, T=1.2$ \\
\hline 0.1 & 0.1 & $7.51 \times 10^{-2}$ & $6 . \times 10^{-2}$ & $5.42 \times 10^{-2}$ & $1.13 \times 10^{-1}$ \\
\hline 0.05 & 0.05 & $4.2 \times 10^{-2}$ & $3.1 \times 10^{-2}$ & $2.8 \times 10^{-2}$ & $5.89 \times 10^{-2}$ \\
\hline 0.025 & 0.025 & $2.07 \times 10^{-2}$ & $1.66 \times 10^{-2}$ & $1.39 \times 10^{-2}$ & $3.53 \times 10^{-2}$ \\
\hline 0.0125 & 0.0125 & $1.13 \times 10^{-2}$ & $8.8 \times 10^{-3}$ & $7.1 \times 10^{-3}$ & $2.16 \times 10^{-2}$ \\
\hline 0.00625 & 0.00625 & $5.7 \times 10^{-3}$ & $4.9 \times 10^{-3}$ & $3.7 \times 10^{-3}$ & $1.55 \times 10^{-2}$ \\
\hline
\end{tabular}

Table 1: $L^{1}$-discrete relative errors for the GD model

\begin{tabular}{|c|c|c|c|c|c|}
\hline$\Delta t$ & $\Delta a$ & $e_{1}^{N_{T}}, T=0.6$ & $e_{2}^{N_{T}}, T=0.6$ & $e_{1}^{N_{T}}, T=1.2$ & $e_{2}^{N_{T}}, T=1.2$ \\
\hline 0.1 & 0.1 & $4.33 \times 10^{-4}$ & $1.22 \times 10^{-2}$ & $1.2 \times 10^{-3}$ & $1.38 \times 10^{-2}$ \\
\hline 0.05 & 0.05 & $2.19 \times 10^{-4}$ & $6.13 \times 10^{-3}$ & $6.05 \times 10^{-4}$ & $6.93 \times 10^{-3}$ \\
\hline 0.025 & 0.025 & $1.1 \times 10^{-4}$ & $3.07 \times 10^{-3}$ & $3.03 \times 10^{-4}$ & $3.47 \times 10^{-3}$ \\
\hline 0.0125 & 0.0125 & $5.56 \times 10^{-5}$ & $1.53 \times 10^{-3}$ & $1.52 \times 10^{-4}$ & $1.73 \times 10^{-3}$ \\
\hline 0.00625 & 0.00625 & $2.76 \times 10^{-5}$ & $7.66 \times 10^{-4}$ & $7.63 \times 10^{-5}$ & $8.67 \times 10^{-4}$ \\
\hline
\end{tabular}

Table 2: $L^{1}$-discrete relative errors for the ID model

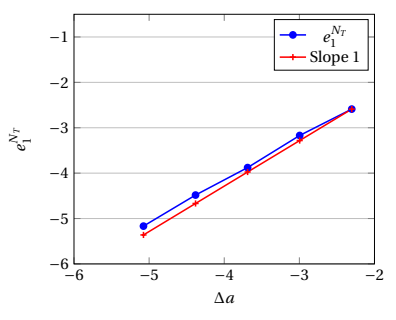

(a) $V_{1}$ at $T=0.6$

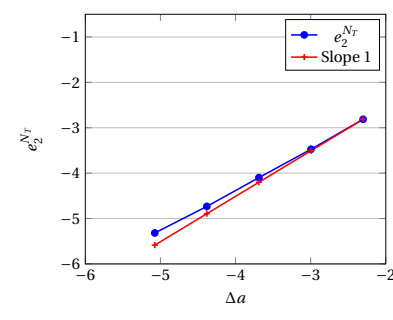

(b) $V_{2}$ at $T=0.6$

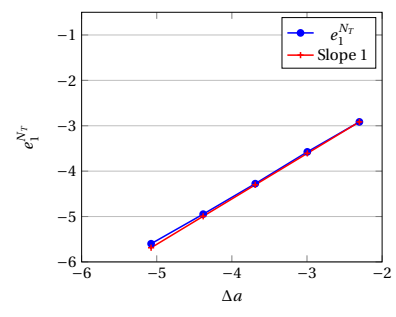

(c) $V_{1}$ at $T=1.2$

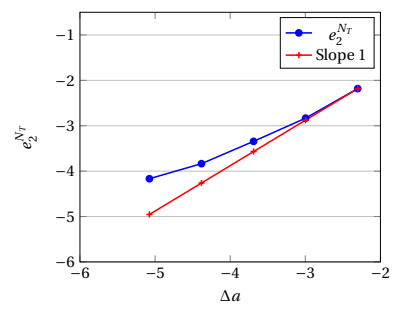

(d) $V_{2}$ at $T=1.2$

Figure 5: $L^{1}$-discrete relative errors for the GD Model in $\log / \log$ scale at $T=0.6$ and $T=1.2$ 


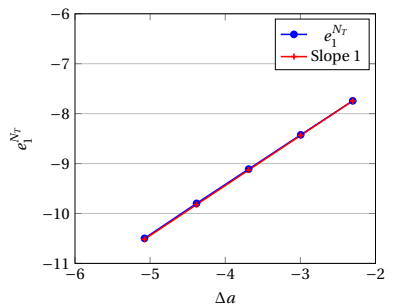

(a) $V_{1}$ at $T=0.6$

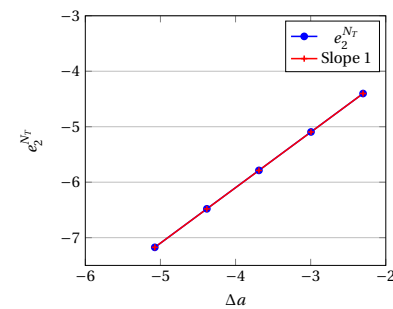

(b) $V_{2}$ at $T=0.6$

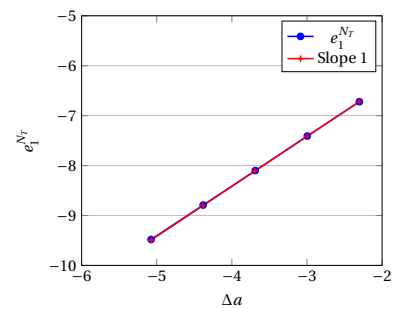

(c) $V_{1}$ at $T=1.2$

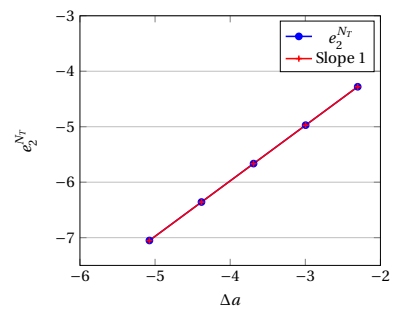

(d) $V_{2}$ at $T=1.2$

Figure 6: $L^{1}$-discrete relative errors for the ID model in $\log / \log$ scale at $T=0.6$ and $T=1.2$

\subsection{A first comparison between the two models}

In this section we consider once again the initial conditions (3.17), (3.18) and we compute the evolution of the vole populations in the two nodes by the GD model when the parameter of minimal time span for a dispersal is reduced to $\eta=3 \times 10^{-3}, c=1$ and the final time is $T=1.3$. The result obtained for $\Delta a=\Delta t=3 \times 10^{-4}$ is in Figures 7.

We can observe that the profile of the solution is, at a first approximation, similar to the solution obtained from the same initial condition by the ID model, see Figure 4. In particular we observe that the two dispersals occurring in the fist cell for $t>1$ concerne a so small number of juveniles that none of them reaches the second cell, so that the dynamics of the second cell is almost identical in the two simulations.

This means that, even if the ID model is necessarily less precise, it suffices to capture the dynamics we would observe in the GD model for small values of $\eta$.

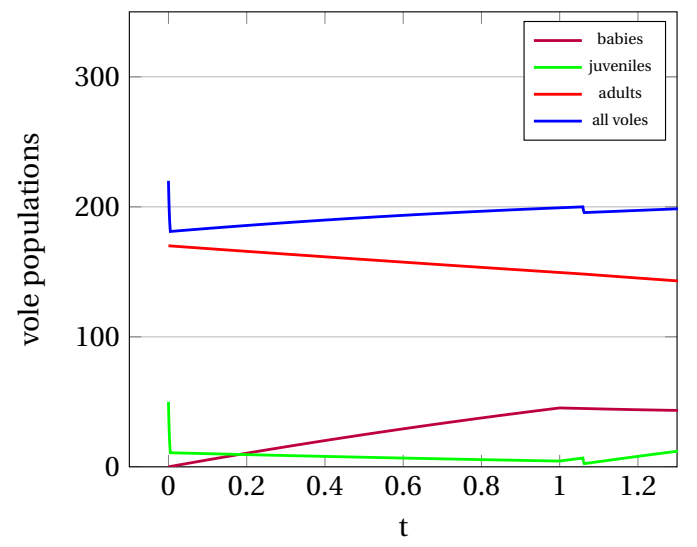

(a) Node $V_{1}$

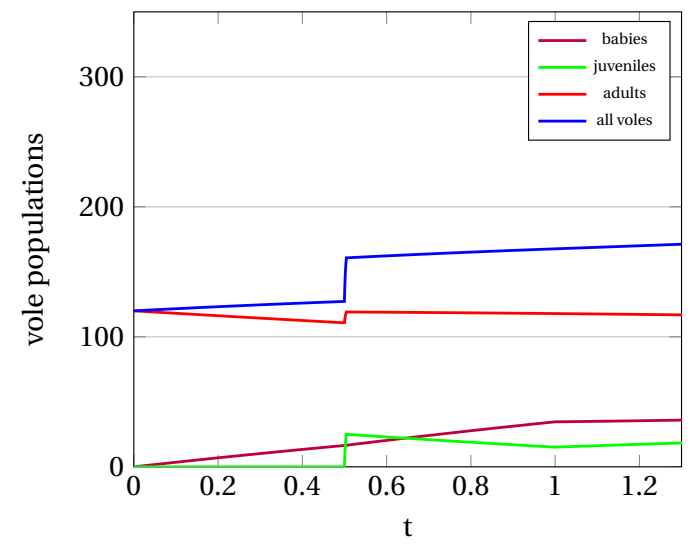

(b) Node $V_{2}$

Figure 7: The voles populations of the two nodes computed with GD model for $\eta=3 \times 10^{-3}$

\section{Numerical simulations}

In this section, we perform numerical simulations on the two models, in order to show their ability to reproduce the qualitative behavior of vole populations and in particular their spatial dynamics. In this paper we limit our attention to simple toy models as the implementation of the schemes on graph representing large regions would require a large effort in optimization and in parallelization of the codes, which we defer to future investigation. 
Everywhere in the following we assume, in the GD model, that $c=1$ and that the minimal duration of a dispersal is $\eta=7 / 18$ that corresponds to one week in real time.

\subsection{Two-nodes dynamics}

In this section, we investigate the evolution of a population of voles on a graph consisting of two nodes assumed to represent two different types of landscapes and the edge linking them. The landscapes we consider are described below:

- The first node represents a crop field, which is a source of abundant food and shelter for voles during summer and spring. This traduces into high reproduction rate and low mortality rate in the warm season. On the contrary mortality becomes high at harvesting time and remains so up to the end of one year cycle.

To reproduce such properties, we consider the reproduction and mortality rate functions defined by (2.9), (2.10) and (2.12) where we assume $\alpha=1, \delta=1$ and $\kappa=2.6$.

- The second node represents a forest, where the population of voles is almost stable over several years. The reproduction rate is lower than in the crop field, but the mortality varies less dramatically from warm to cold season.

To reproduce such properties, we consider the reproduction and mortality rate functions defined by (2.9), (2.10) and (2.12) where we assume $\alpha=0.656, \delta=1$ and $\kappa=1.5$.

We assume that the travel time from one node to the other is $\ell=0.5$. Then we consider the travel time matrix and the distribution matrix as

$$
\mathscr{L}=\left(\begin{array}{cc}
0 & 0.5 \\
0.5 & 0
\end{array}\right) \quad \text { and } \quad \mathbf{K}=\left(\begin{array}{ll}
0 & 1 \\
1 & 0
\end{array}\right)
$$

respectively. The initial conditions are of 70 adult individuals in each node, equidistributed over their age class. The simulations, performed with $\Delta a=\Delta t=0.00625$, are depicted in Figures 8.

Both models reproduce the cyclic behavior of the population. A characteristic feature of the ID model is the appearance of sharp peaks, as in Figure 8(d). This is due to the fact that voles coming from the node representing the crop field reach their destination at moments where the total population in the node representing forest is close to the dispersal threshold. This sudden population increase, sufficient to trigger dispersal, lasts only one time step. On the contrary, no peaks arise in Figure 8(c) as in the GD model, dispersals and arrivals happen progressively. Moreover, since not all of the juveniles leave, the variation in the total population is lower, see Figures 8(a) and 8(c).

\subsection{Three-nodes dynamics}

In this section we run our models on a complete graph with three nodes, $V_{i}, i=1,2,3$. This allows us to use a more interesting distribution matrix to reproduce, for example, the fact that voles prefer to move downhill rather than uphill, and that they are more likely to stop at places where resources are more abundant. Since the availability of food may change in time, we consider a time dependent distribution matrix in our second example.

The simulations of this section are performed with $\Delta a=\Delta t=0.0125$. 


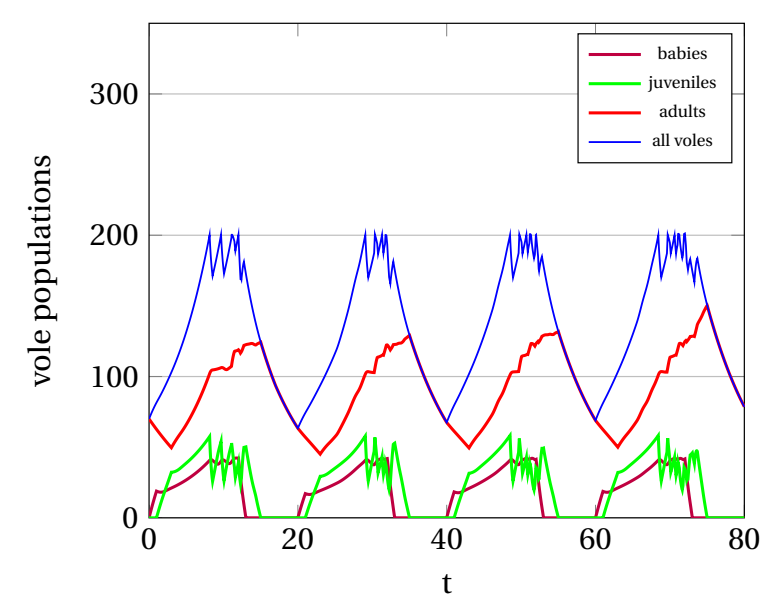

(a) Crop field, GD model

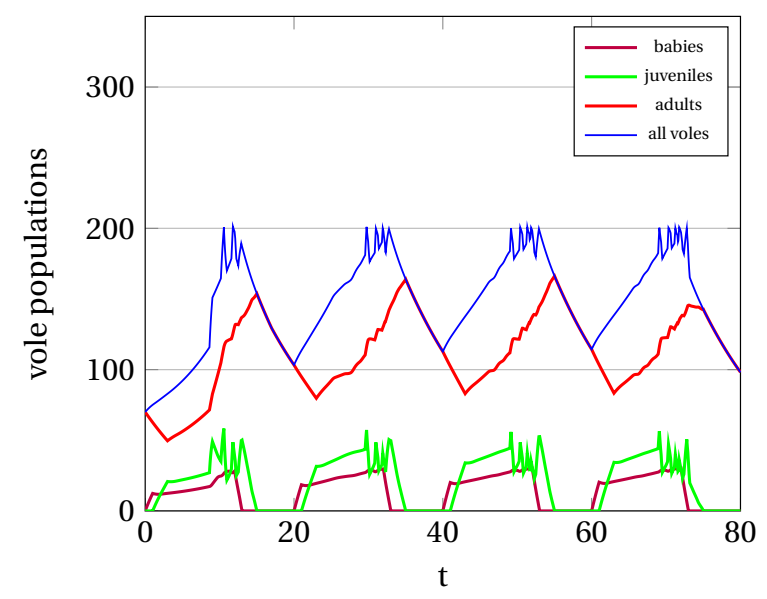

(c) Forest, GD model

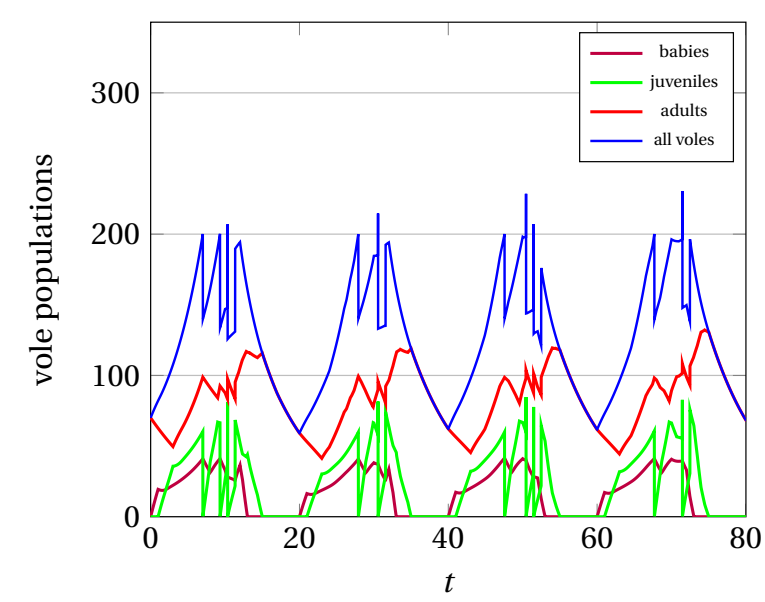

(b) Crop field, ID model

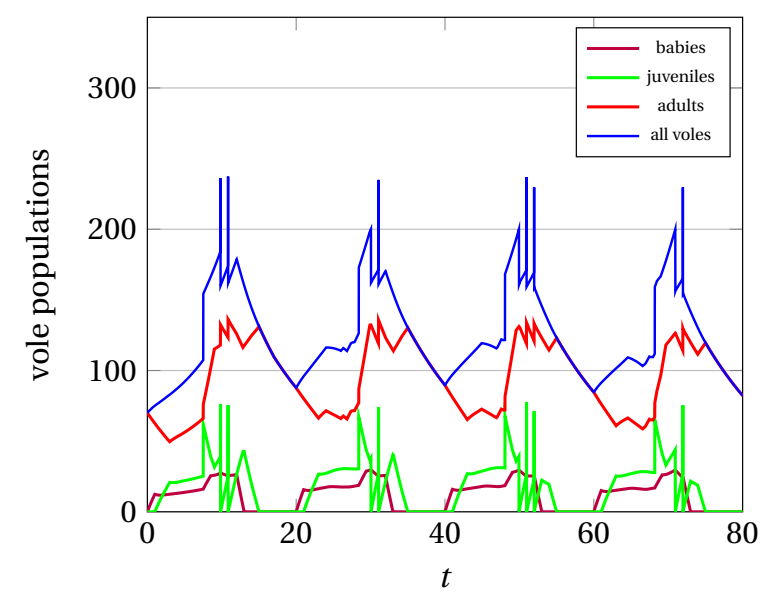

(d) Forest, ID model

Figure 8: With reference to subsection 4.1: the evolution of the population over 4 years for the two models.

\subsubsection{Scenario 1: Three nodes located at different heights}

We assume here that the node $V_{1}$ is located at the highest spot and $V_{3}$ at the lowest one. For all the nodes, we use the mortality and the reproduction rates defined by (2.9), (2.10) and (2.12) with $\alpha=\delta=1$ and $\kappa=1.5$. As initial conditions we take $\phi_{1}(0)=100, \phi_{2}(0)=50$ equidistributed over the adult age class, and $\phi_{3}(0)=0$, that means that the third node is initially empty. It has been observed that when dispersal occur, voles move more frequently downhill (2 times out of 3), see for instance $[5,13]$. In other to take into account this characteristic, we consider the following distribution matrix

$$
\mathbf{K}=\left(\begin{array}{ccc}
0 & 1 & 0 \\
1 / 3 & 0 & 2 / 3 \\
0 & 1 & 0
\end{array}\right)
$$


In particular, this means that all the dispersals taking place from $V_{1}$ or $V_{3}$ head toward $V_{2}$, while 2 out of 3 voles leaving $V_{2}$ would go to $V_{3}$. We fix the travel times matrix as

$$
\mathscr{L}=\left(\begin{array}{ccc}
0 & 1 & 1.5 \\
1 & 0 & 0.5 \\
1.5 & 0.5 & 0
\end{array}\right)
$$

Figures 9 show the evolution of the population for both models over three years. We can observe numerous outbreaks from $V_{2}$, which are due to the fact that all the voles that disperse from $V_{1}$ and $V_{3}$ arrive in this node. Moreover from Figure 9 (c), we can see that, due to the relatively long duration of dispersal, the total population in $V_{2}$ largely exceeds the threshold 200 in the second and third year.

We can also notice that a persistent colony appears in $V_{3}$ thanks to the individuals arriving from the other nodes.

\subsubsection{Scenario 2: Time dependent distribution matrix}

We consider a domain partitioned into three cells. The first one, $V_{1}$, is located at a higher elevation than the two others, which are on the same level. We make the following choice of parameters :

- In $V_{1}, \alpha=\delta=1, \kappa=1.5$ and the initial condition consists of 100 adults individuals equidistributed over their age class.

- In $V_{2}$, we imagine to have a crop field as in Section 4.1. Then we set $\alpha=\delta=1, \kappa=2.6$ and the initial condition consists of 70 adults individuals equidistributed over their age class.

- In $V_{3}$, we have a forest as in Section 4.1. Then we set $\alpha=0.656, \delta=1, \kappa=1.5$ and take the same initial condition as in $V_{2}$.

We assume now that during spring and summer, i.e. for $t \in[0,10]$, eventual dispersers tend to move to the second colony because of the abundance of food source in a crop landscape. While in autumn and winter, i.e. for $t \in[10,20]$, they rather move downhill to the third cell, as we mentioned in Section 4.1. Therefore we consider the dispersal ratio matrix $\mathbf{K}_{1}$ for $t \in[0,10]$ and $\mathbf{K}_{2}$ for $t \in[10,20]$,

$$
\mathbf{K}_{1}=\left(\begin{array}{ccc}
0 & 0.7 & 0.3 \\
0.3 & 0 & 0.7 \\
0.2 & 0.8 & 0
\end{array}\right) \quad, \quad \mathbf{K}_{2}=\left(\begin{array}{ccc}
0 & 0.3 & 0.7 \\
0.3 & 0 & 0.7 \\
0.3 & 0.7 & 0
\end{array}\right) .
$$

We assume that the travel time between any two nodes is 0.5 , so that

$$
\mathscr{L}=\left(\begin{array}{ccc}
0 & 0.5 & 0.5 \\
0.5 & 0 & 0.5 \\
0.5 & 0.5 & 0
\end{array}\right)
$$

In Figures 10 we present the evolution of the populations of voles in the three nodes for both models during three years. We can observe several dispersals occuring every year from each node. As we already noticed in previous simulations, the arrivals of voles increase the frequency of outbreaks. Moreover, we can see that in $V_{2}$, that is the crop field landscape, the population of voles increases over the reproduction season with the appearance of peaks for the second model in Figure 10(d). However, due to the high mortality rate after harvesting, the increase of the total population each year is slow and contained, in particular its minimal value in a one year period is almost constant. On 


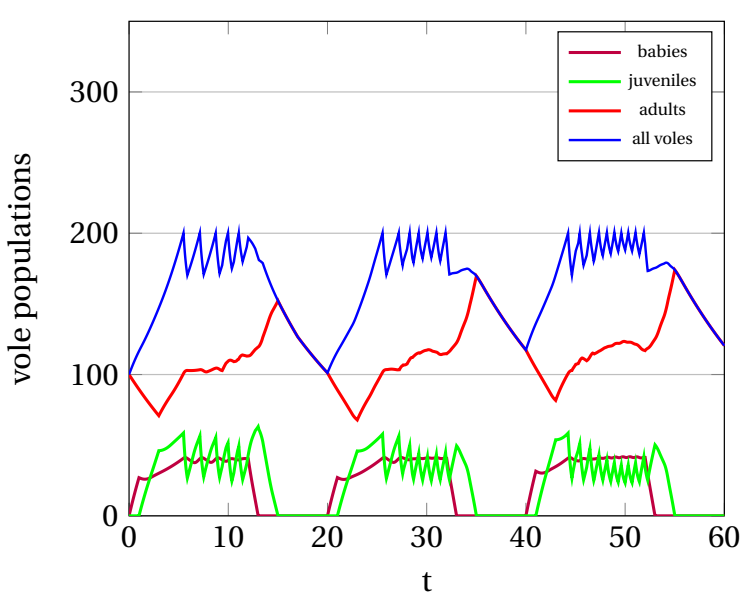

(a) $V_{1}$, GD model

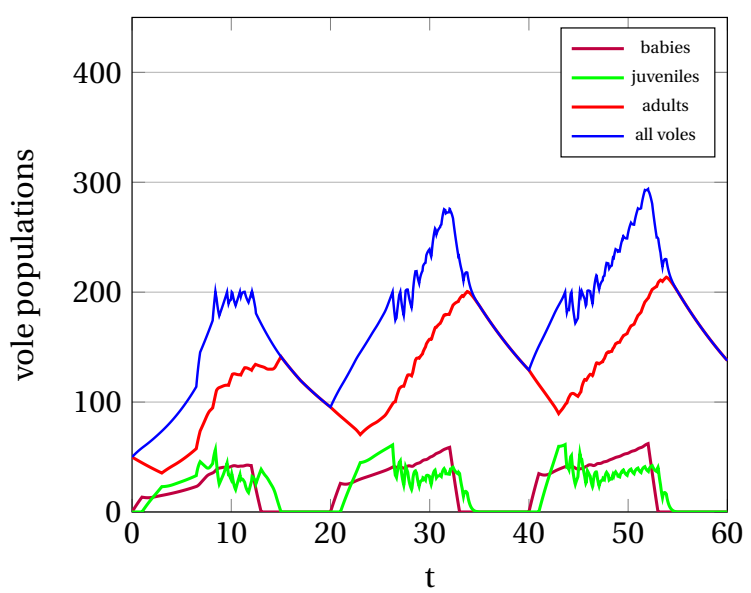

(c) $V_{2}$, GD model

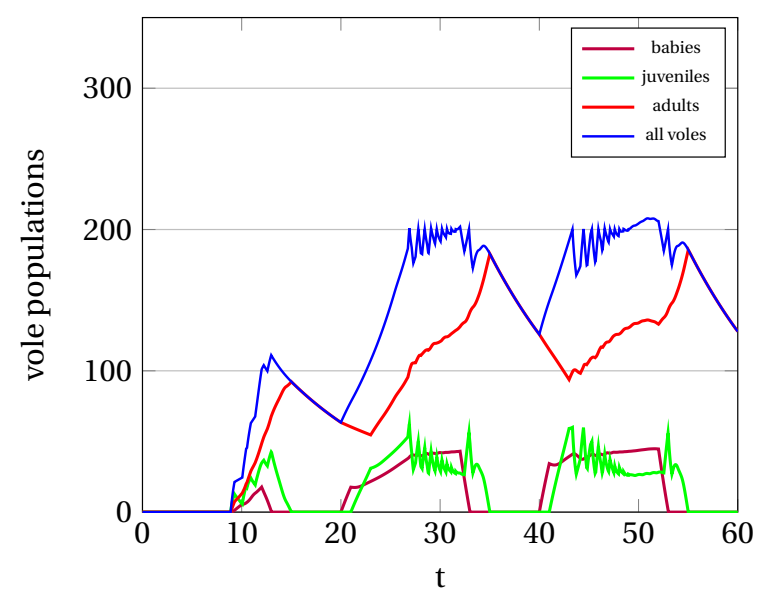

(e) $V_{3}$, GD model

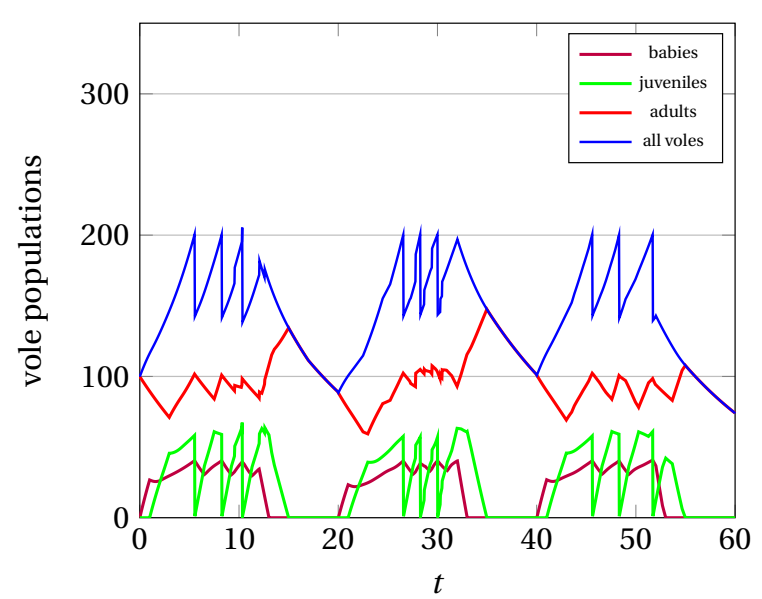

(b) $V_{1}$, ID model

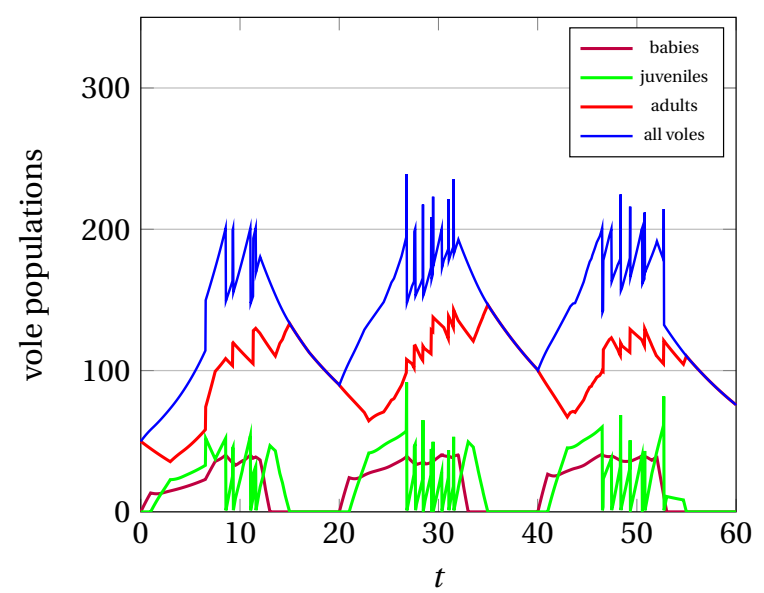

(d) $V_{2}$, ID model

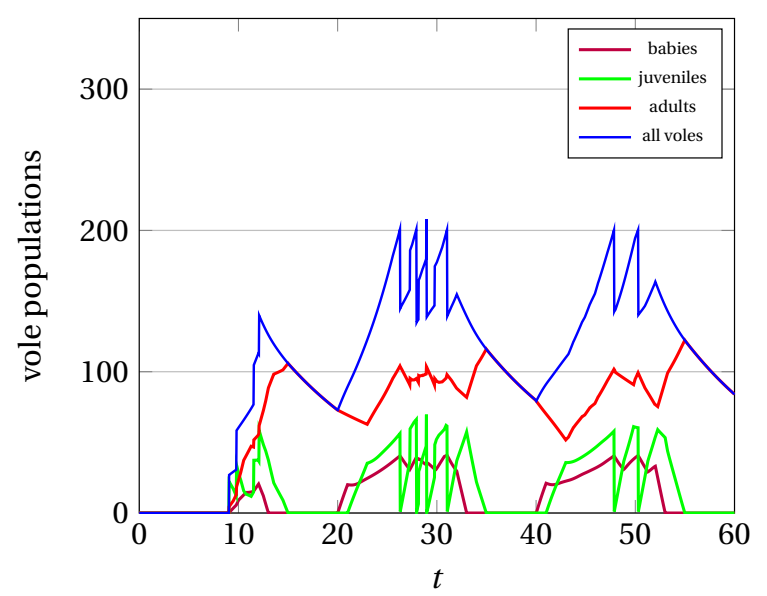

(f) $V_{3}$, ID model

Figure 9: With reference to Section 4.2.1: The population of voles over 3 years in $V_{1}, V_{2}$ and $V_{3}$ for both models.

the contrary, in $V_{3}$, the minimal value of the total population increases over one year, which means that there are more and more adults. At the end of the third year, we have approximatively 110 and 96 adult individuals in Figure $10(\mathrm{e})$ 
and Figure 10(f) respectively. As a consequence of the increment of the adult population, we can see in the two figures that there are more and more dispersal outbreaks. Figure 10(f) also shows more peaks than in Figure 10(d).

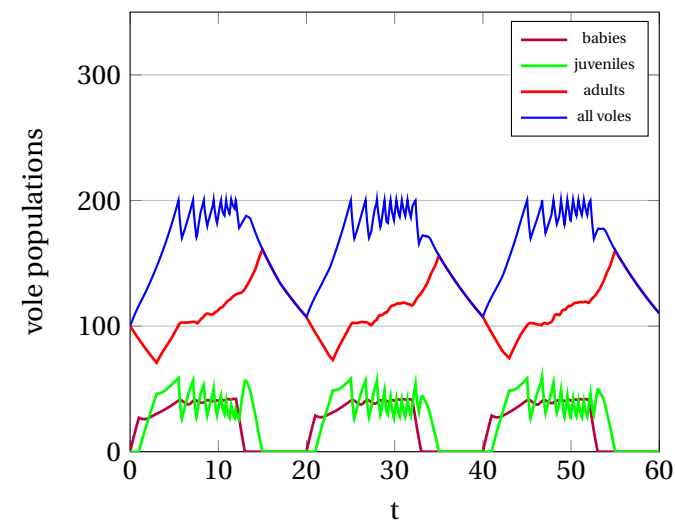

(a) $V_{1}$, GD model

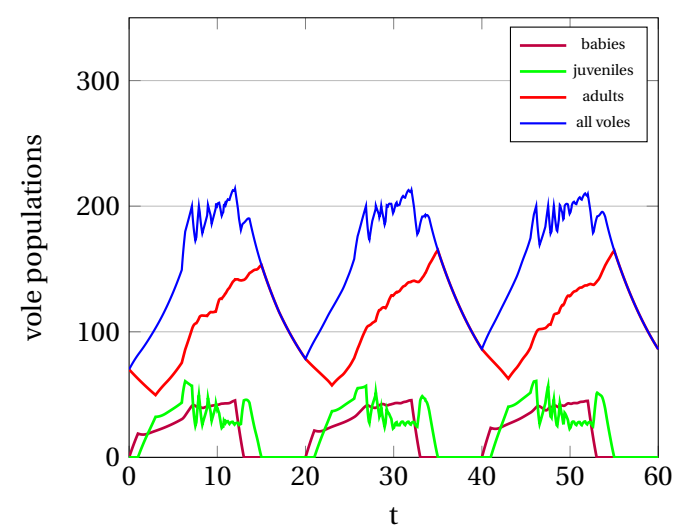

(c) $V_{2}$, GD model

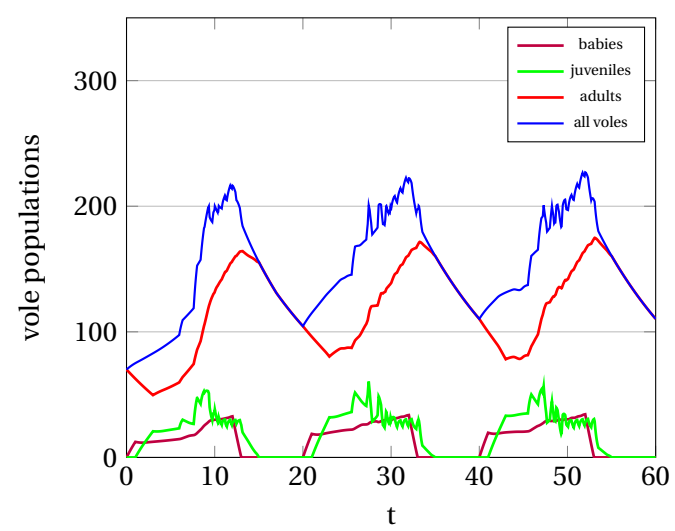

(e) $V_{3}$, GD model

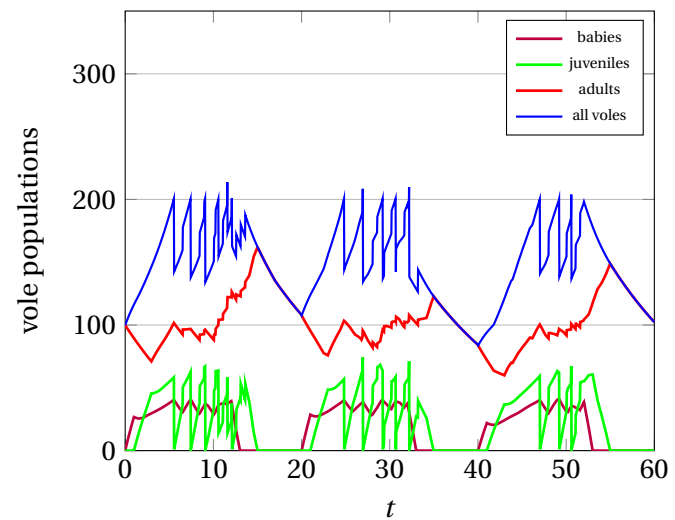

(b) $V_{1}$, ID model

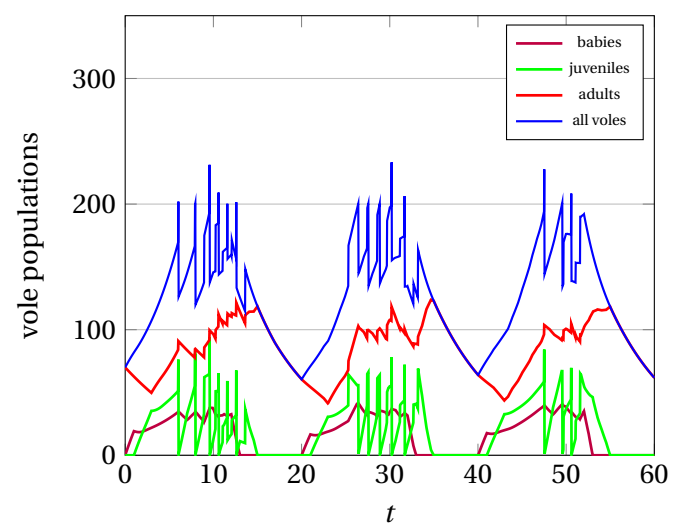

(d) $V_{2}$, ID model

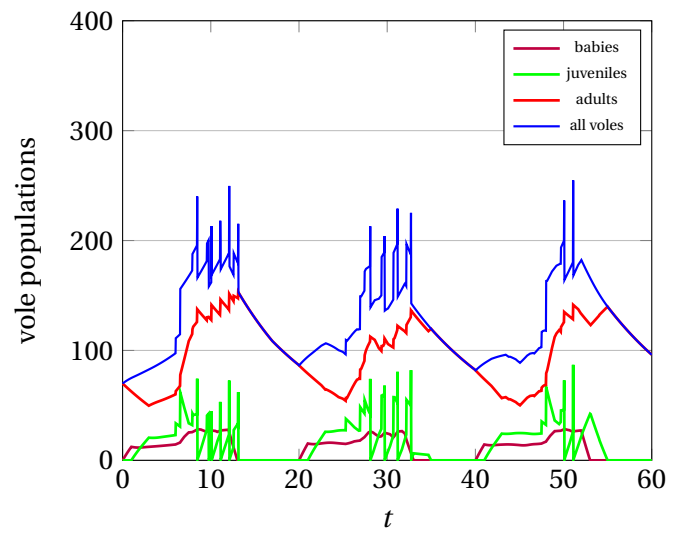

(f) $V_{3}$, ID model

Figure 10: With reference to Section 4.2.2: the population of voles over 3 years on three nodes representing different landscapes. 


\section{Conclusions}

In this paper, we propose two models to describe the spatial dynamic of voles populations. The models are based on age-structured transport equations set on a graph. The nodes of the graph represent uniform landscape areas of relatively small size, where voles can settle. Edges are used to model the main feature of voles' macroscopic spatial dynamics, which is the dispersal of juvenile individuals whenever the total population at one node reaches a threshold number. This is supposed to be a gradual process in the first model while it takes place instantaneously in the second model. The parameters in the reproduction and mortality rates allows to reproduce the evolution in different landscapes.

The numerical simulations that we presented show the ability of the models to reproduce cycles of dispersals in the populations of voles, as well as persistence or extinction of a colony. The results that we obtained in this paper allow us to pursue the work with the construction of a hybrid model combining the ID model to describe the voles population dynamics at the nodes and the agents-based model developed in [13] to reproduce the spatial dynamics on large graphs. In a different direction it is also possible to enhance the two models by considering a distribution matrix which is not given a priori, but depends on the current state of the system. For example, one could imagine that dispersers would avoid an already overcrowded node.

\section{Appendices}

\section{A Allee's effect : survival number of a colony}

We investigate in this part the minimal number of juvenile or adult individuals needed to maintain the existence of a colony when we consider the reproduction and the mortality rates defined by (2.9), (2.10) and (2.12) with $\alpha=\delta=1$ and $\kappa=1.5$. To that end, in the boundary condition at $a=0$, (2.6), we consider a non constant function $\mathscr{A}$ of the form

$$
\mathscr{A}(\phi)=\frac{\beta \phi^{\gamma}}{(B+\phi)^{\gamma}}
$$

so that the model can reproduce the Allee's effect. For simplicity, we just consider case in which $\beta=1, \gamma \in \mathbb{N}$, and

$$
B=\left(\frac{\gamma+1}{\gamma-1}\right) \phi_{*}^{\gamma}
$$

where $\phi_{*}$ is a given value. As an example, if we take $\gamma=8$ and $\phi_{*}=20$ we obtain

$$
\mathscr{A}(\phi)=\frac{\phi^{8}}{\left(\frac{9.20^{8}}{7}+\phi^{8}\right)}, \quad \phi \geq 0 .
$$

This function has an inflection point at $\phi=20$ whose value is $\mathscr{A}(20)=7 / 16=0.4375$, see Figure 11 . This factor makes the reproduction rate almost insignificant when the total population is less than 20 . Considering one node and using the mortality and the reproduction rates defined by (2.9), (2.10) and (2.12) with $\alpha=\delta=1$ and $\kappa=1.5$, that is the same parameters as in Figures 2(a) and 2(b), we can see in Figures 12, that we need at least 19 juvenile individuals or 20 adult individuals in the initial condition for the colony to persist over several years without any external support. Figures 13 show the evolution of the populations of voles inside a colony during two years when we start from 18 juvenile and 19 adult individuals respectively. As we can see, the colony disappears in less than two years. We can calibrate the model to obtain a realistic survival number by changing the inflection point of the function $\mathscr{A}$. 


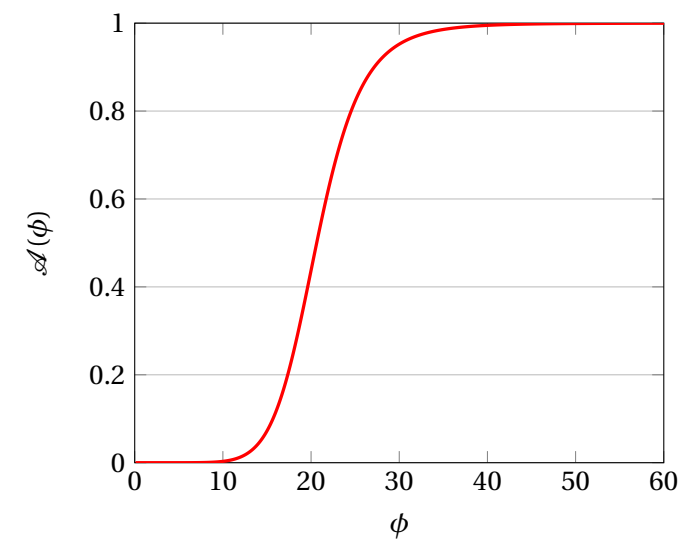

Figure 11: The function defined in (A.36).
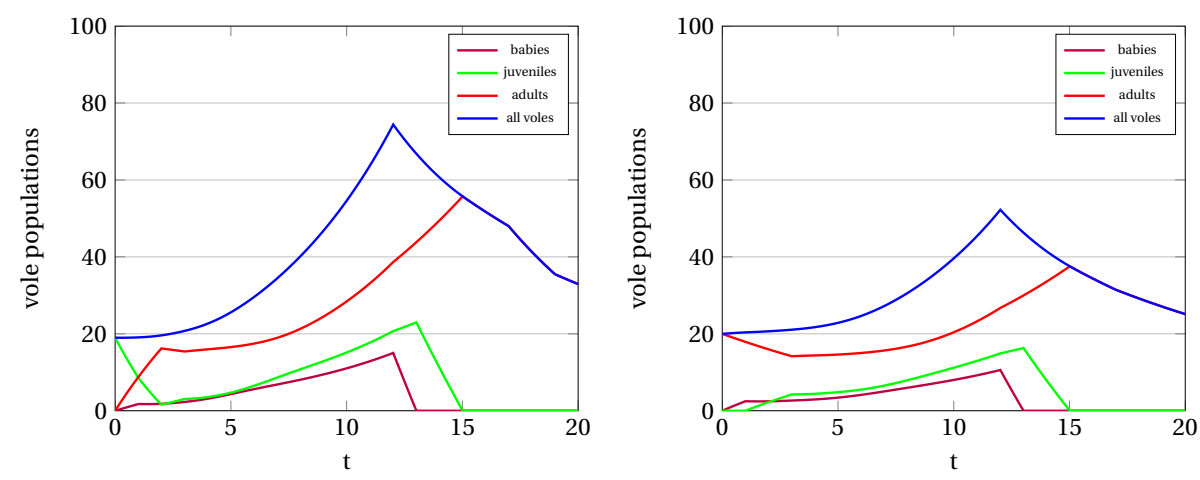

Figure 12: Populations of voles in a colony starting with 19 juvenile (left) or 20 adult (right) individuals.
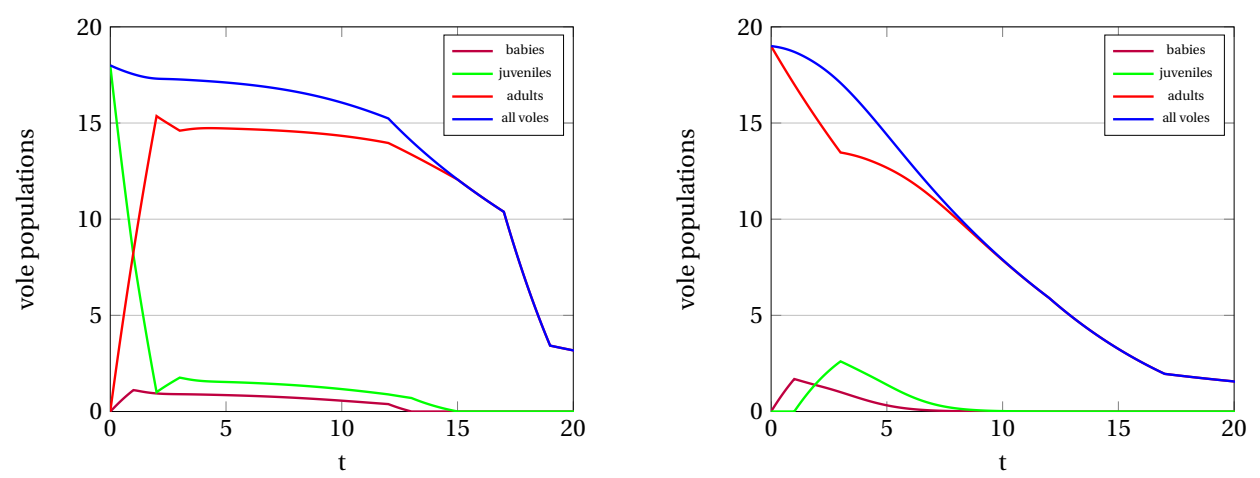

Figure 13: Populations of voles in a colony starting with 18 juvenile (left) or 19 adult (right) individuals.

\section{Acknowledgments}

All the authors acknowledge the support of the Région Bourgogne Franche-Comté, projet 2017-2020 "Analyse mathématique et simulation numérique d'EDP issus de problèmes de contrôle et du trafic routier" and of the Zone Atelier Arc Jurassien projet 2018 "Modélisation et simulations numériques de la dynamique spatiale des populations de campagnols". 


\section{References}

[1] H. P. Andreassen, P. Glorvigen, A. Rémy, and R. A. Ims. New views on how population-intrinsic and communityextrinsic processes interact during the vole population cycles. Oikos, 122(4):507-515, 2013.

[2] K Berthier, S Piry, J.-F. Cosson, P. Giraudoux, J.-C. Foltête, R. Defaut, D. Truchetet, and X. Lambin. Dispersal, landscape and travelling waves in cyclic vole populations. Ecology Letters, 17(1):53-64, 2014.

[3] D. Cerqueira, B. De Sousa, C. Gabrion, P. Giraudoux, J.P. Quéré, and P. Delattre. Cyclic changes in the population structure and reproductive pattern of the water vole, arvicola terrestris linnaeus, 1758. Mammalian Biology, 71(4):193 - 202, 2006.

[4] G. M. Coclite, C. Donadello, and T. N. T. Nguyen. A PDE model for the spatial dynamics of a voles population structured in age. Nonlinear Anal., 196:111805, 26, 2020.

[5] P. Delattre, B. De Sousa, and et al E. Fichet-Calvet. Vole outbreaks in a landscape context: evidence from a six year study of microtus arvalis. Landscape Ecology, 14:401-412, 1999.

[6] L. C. Evans. Partial differential equations, volume 19 of Graduate Studies in Mathematics. American Mathematical Society, Providence, RI, 1998.

[7] P. Giraudoux, P. Delattre, M. Habert, J. Quéré, S. Deblay, R. Duhamel R. Defaut, M. Moissenet, D. Salvi, and D. Truchetet. Population dynamics of fosserial water vole (arvicola terrestris scherman) : a land use and a landscape perspective. Agriculture, Ecosystems and Environment, 66(1):47-60, 1997.

[8] P. Giraudoux, P. Villette, J. Quéré, and et al. Weather influences m. arvalis reproduction but not population dynamics in a 17-year time series. Sci Rep, 9:13942, 2019.

[9] R. Ims and H. Andreassen. Spatial synchronization of vole population dynamics by predators birds. Nature, 408:194-196, 2000.

[10] D. M. Johnson, U. Büntgen, D. C. Frank, K. Kausrud, K. J. Haynes, A. M. Liebhold, J. Esper, and N. Chr. Stenseth. Climatic warming disrupts recurrent alpine insect outbreaks. Proc. Natl. Acad. Sci., 107(47):20576-20581, 2010.

[11] C. Krebs. Population fluctuations in rodents. The University of Chicago Press, Chicago and London, 2013.

[12] R. J. LeVeque. Finite volume methods for hyperbolic problems. Cambridge Texts in Applied Mathematics. Cambridge University Press, Cambridge, 2002.

[13] N. Marilleau, C. Lang, and P. Giraudoux. Coupling agent-based with equation-based models to study spatially explicit megapopulation dynamics. Ecological Modelling, 384:34 - 42, 2018.

[14] J.D. Murray. Mathematical Biology I, An introduction. Interdisciplinary applied mathematics. Springer, third edition edition, 2004.

[15] M. Pascal and T. Boujard. Essai de typologie de paramètres démographiques et morphologiques de la fraction colonisatrice d'une population de campagnols terrestres (Arvicola terrestris scherman). Terre et Vie, 42:357-376, 1987.

[16] B. Perthame. Transport equations in biology. Birkhäuser, 2007. 
[17] J. Quéré and H. Le Louarn. Les rongeurs de France. Editions QUAE, Versailles, 2011.

[18] F. Saucy and B. Schneiter. Juvenile dispersal in the vole arvicola terrestris during rainy nights : a preliminary report. Bull. Soc. Vaud. Sc. Nat., 84(4):333-345, 1997.

[19] T. N. Sherratt, X. Lambin, S. J. Petty, J. L. Mackinnon, C.F. Coles, and C. J. Thomas. Use of coupled oscillator models to understand synchrony and travelling waves in populations of the field vole microtus agrestis in northern england. J. Appl. Ecol., 37(s1):148-158, 2000.

[20] H. R. Thieme. Mathematics in Population Biology. Princeton Series in Theoretical and Computational Biology. Princeton, 2003.

[21] G. F. Webb. Population models structured by age, size, and spatial position. In Structured population models in biology and epidemiology, volume 1936 of Lecture Notes in Math., pages 1-49. Springer, Berlin, 2008. 